\title{
SPEKTRALFUNKTIONEN EINER KLASSE VON DIFFERENTIALOPERATOREN ZWEITER ORDNUNG MIT NICHTLINEAREM EIGENWERTPAR AMETER
}

\author{
HEINZ LANGER
}

Bekanntlich ([3], §13) ist das Stieltjessche Momentenproblem äquivalent mit der Aufgabe, alle Spektralfunktionen mit nichtnegativem Träger einer Stieltjesschen Saite, d. h., einer Anfangswertaufgabe der Form

$$
\begin{aligned}
d f_{+}^{\prime}(x)+\lambda f(x) d M(x) & =0, \quad 0 \leqq x \leqq l, \\
f_{-}^{\prime}(0) & =0
\end{aligned}
$$

zu bestimmen ( $M=$ nichtabnehmende Treppenfunktion). Das indefinite Analogon des Stieltjesschen Momentenproblems (das Problem $P\left(\boldsymbol{H}_{\varkappa}^{+}\right)$ in der Terminologie von [6]) läßt sich entsprechend auf die Bestimmung aller Spektralfunktionen einer Stieltjesschen Saite vom Index $\varkappa$, die Dipole und negative Massen tragen kann, zurückführen. Unter einer solchen Saite verstehen wir eine Anfangswertaufgabe der Form

$$
\begin{gathered}
d f_{+}^{\prime}(x)+\lambda f(x) d M(x)+\lambda^{2} f(x) d D(x)=0, \quad 0 \leqq x \leqq l, \\
f_{-}^{\prime}(0)=0
\end{gathered}
$$

mit zwei Treppenfunktionen $M$ und $D$, deren Sprungstellen sich höchstens bei $l$ häufen; dabei ist die Funktion $D$ nichtfallend und die Anzahl derjenigen Punkte $x$, in denen $D(x+0)-D(x-0)>0$ oder $M(x+0)-$ $M(x-0)<0$ gilt, endlich und zwar gleich $x$.

In der vorliegenden Arbeit untersuchen wir die Anfangswertaufgabe (0.2) under der Voraussetzung, daß $M$ eine Funktion von beschränkter Variation und $D$ eine beschränkte nichtfallende Funktion auf dem kompakten Intervall $[0, l]$ der reellen Achse sind. Nach vorbereitenden Betrachtungen führen wir in $\S 1$ den Weylschen Koeffizienten $m_{N}$ der Aufgabe (0.2) ein, der zu einer Randbedingung der Form

$$
f_{-}^{\prime}(l) N(\lambda)+f(l)=0
$$


am rechten Endpunkt gehört. Die Anfangswertaufgabe (0.2) läßt sich auf eine Anfangswertaufgabe für ein kanonisches System von positivem Typ zurückführen, dessen Spektralfunktionen wir als die Spektralfunktionen der Aufgabe (0.2) definieren. Aus Ergebnissen von [3], § 14, folgt dann leicht eine Beschreibung aller dieser Spektralfunktionen. Sie entsprechen genau denjenigen Randbedingungen (0.3) am rechten Endpunkt, für welche die Funktion $\lambda \rightarrow \lambda m_{N}(\lambda)$ zur Nevanlinnaklasse $N_{0}$ gehört.

Bezeichnet $V$ die Funktion $V(x):=|M|(x)+D(x) \quad(|M|(x)=$ Totalvariation von $M$ auf $[0, x])$, so kann man der Anfangswertaufgabe (0.2) eine bezüglich des Eigenwertparameters quadratische Operatorenschar im Raume $L_{V}^{2}$ zuordnen. Nach Übergang zu einem bezüglich des Eigenwertparameters linearen Problem (im Raume $L_{V}^{2} \oplus L_{V}^{2}$ ) lassen sich z. B. mit Hilfe der Methode der richtenden Funktionale von M.G. Krĕn ebenfalls Spektralfunktionen für die Aufgabe (0.2) einführen. In $\S 3$ zeigen wir, daß diese Spektralfunktionen mit den in $\S 1$ definierten übereinstimmen. Ist insbesondere $D \equiv 0$, so fallen sie mit den $A$-Spektralfunktionen aus [8], § 3 , zusammen.

In $\S 2$ betrachten wir als Anwendung der Ergebnisse von 11 die SturmLiouvillesche Anfangswertaufgabe

$$
\begin{gathered}
-\left(p(x) y^{\prime}(x)\right)^{\prime}+q(x) y(x)=\lambda r(x) y(x), \quad 0 \leqq x \leqq b, \\
p(0) y^{\prime}(0) \sin \alpha-y(0) \cos \alpha=0
\end{gathered}
$$

mit indefiniter Gewichtsfunktion $r$ und $q \geqq 0$ (vgl. [2]). Analog wie dieses Problem im Falle einer nichtnegativen Gewichtsfunktion in [3], § 14, auf eine Aufgabe der Form (0.1) zurückgeführt wurde, ergibt sich hier für gewisse Anfangsbedingungen im Nullpunkt eine Aufgabe (0.1) mit einer nichtmonotonen Funktion $M$ (oder (0.2) mit $D \equiv 0)$. Die betrachteten Spektralfunktionen entsprechen hier denjenigen Randbedingungen (0.3) am rechten Endpunkt, für die mit dem Weylschen Koeffizienten $l_{N}^{(\alpha)}$ (siehe (2.12)) die Funktion $\lambda \rightarrow \lambda\left(l_{N}^{(\alpha)}(\lambda)+\cot \alpha\right)$ zur Klasse $N_{0}$ gehört.

In der vorliegenden Arbeit beschränken wir uns (mit einer Ausnahme am Ende von § 3, wo wir eine in [2] aufgeworfene Frage beantworten) auf den sog. regulären Fall, jedoch lassen sich einige Ergebnisse (insbesondere der Abschnitte 1.4, 2.3 und des § 3) unmittelbar auf den singulären Fall übertragen. Ausführlicher werden wir auf singuläre Operatoren der Form (0.2) an anderer Stelle eingehen.

Im Zusammenhang mit den hier betrachteten Problemen spielen die Funktionen der von Rolf Nevanlinna in [9] eingeführten Klasse $N_{0}$ sowie der von M.G. KreǏn und dem Verfasser in [6] eingeführten Klassen $N_{x}$ eine wesentliche Rolle. Obwohl wir in dieser Arbeit die Hilfsmittel und Methoden aus der Theorie der linearen Operatoren in Räumen mit inde- 
finitem Skalarprodukt nicht explizit benutzen, sei bemerkt, daß die in $\S 3$ eingeführten Operatoren $\boldsymbol{L}$ symmetrisch bezüglich eines indefiniten Skalarproduktes $[\cdot, \cdot]$ sind, genauer, es gilt $[\boldsymbol{L} \boldsymbol{f}, \boldsymbol{f}] \geqq 0$ für alle $\boldsymbol{f} \in \mathfrak{D}(\boldsymbol{L})$. Deshalb hängen diese Untersuchungen eng mit der im Anschluß an die Arbeiten [10]-[14] Rolf Nevanlinnas von E. Pesonen [15] unter etwas einschränkenden Voraussetzungen betrachteten abstrakten Operatoren und ihren Spektralfunktionen zusammen.

Schließlich sei vermerkt, daß die zu den Spektralfunktionen in den $\S \S 2$ und 3 gehörigen Randbedingungen dem sog. S-positiven Fall von $\AA$. Pleijel (siehe z. B. [16]) entsprechen.

\section{Weylscher Koeffizient und Spektralfunktionen der Anfangswertaufgabe (0.2)}

1.1. Auf dem kompakten Intervall $[0, l]$ der reellen Achse seien eine linksseitig stetige, nichtabnehmende beschränkte Funktion $D$ und eine linksseitig stetige Funktion $M$ von beschränkter Variation mit $M(0)=D(0)=0$ gegeben. Mit $|M|(x)$ bezeichnen wir die Totalvariation der Funktion $M$ auf dem Intervall $[0, x]$ und setzen $M_{ \pm}(x):=$ $\frac{1}{2}(|M|(x) \pm M(x)), \quad V(x):=|M|(x)+D(x), \quad 0 \leqq x \leqq l$. Wir setzen außerdem der Einfachheit halber voraus, daß die Randpunkte 0 und $l$ Wachstumspunkte von $V$ sind.

Die Funktionen $M$ und $D$ sind absolutstetig bezüglich $V$; wir benötigen später die zugehörigen $V$-fast-überall bestimmten Dichten, die wir mit $\beta$ und $\gamma$ bezeichnen:

$$
d M(x)=\beta(x) d V(x), \quad d D(x)=\gamma(x) d V(x) .
$$

Dann gilt offensichtlich

$$
|\beta(x)|+\gamma(x)=1 \quad(V \text {-fast-überall }) .
$$

Es sei weiter $\mathfrak{D}_{0}$ die (lineare) Menge aller Funktionen $f$, die sich in der Form

$$
f(x)=f_{0}+f_{0}^{\prime} x+\int_{0}^{x}(x-s) \varphi(s) d V(s)
$$

mit einer Funktion $\varphi \in L^{2}{ }_{V}$ und komplexen Zahlen $f_{0}, f_{0}^{\prime}$ darstellen lassen. Für $f \in \mathbb{D}_{0}$ gilt offensichtlich $f(0)=f_{0}$; wir setzen noch $f_{-}^{\prime}(0):=f_{0}^{\prime}$. Jede Funktion $f \in \mathscr{D}_{0}$ ist absolutstetig auf $[0, l]$, hat in jedem Punkte $x \in[0, l)$ eine rechtsseitige Ableitung 


$$
f_{+}^{\prime}(x)=f_{0}^{\prime}+\int_{0}^{x+0} \varphi(s) d V(s)
$$

und in jedem Punkte $x \in(0, l]$ eine linksseitige Ableitung

$$
f_{-}^{\prime}(x)=f_{0}^{\prime}+\int_{0}^{x-0} \varphi(s) d V(s) .
$$

Für eine $V$-summierbare Funktion $g$ verstehen wir unter einer Lösung $f$ der Differentialgleichung

$$
d f_{+}^{\prime}(x)+\lambda f(x) d M(x)+\lambda^{2} f(x) d D(x)=g(x) d V(x), \quad 0 \leqq x \leqq l,
$$

eine Lösung der Integralgleichung

(1.5) $f(x)=f_{0}+x f_{0}^{\prime}-\int_{0}^{x}(x-s)\left[f(s)\left(\lambda d M(s)+\lambda^{2} d D(s)\right)-g(s) d V(s)\right]$

$\left(f_{0}, f_{0}^{\prime}=\right.$ beliebige komplexe Zahlen), und nennen die Lösung $f$ von (1.5) auch eine Lösung der Anfangswertaufgabe

$$
\begin{gathered}
d f_{+}^{\prime}(x)+\lambda f(x) d M(x)+\lambda^{2} f(x) d D(x)=g(x) d V(x), \quad 0 \leqq x \leqq l, \\
f(0)=f_{0}, \quad f_{-}^{\prime}(0)=f_{0}^{\prime} .
\end{gathered}
$$

Ist nur eine Anfangsbedingung, z. B. $f_{-}^{\prime}(0)=0$, gegeben, so nennen wir jede Lösung $f$ der Integralgleichung (1.5) mit $f_{0}^{\prime}=0, f_{0}$ beliebig, eine Lösung der Anfangswertaufgabe

$$
\begin{gathered}
d f_{+}^{\prime}(x)+\lambda f(x) d M(x)+\lambda^{2} f(x) d D(x)=g(x) d V(x), \quad 0 \leqq x \leqq l, \\
f_{-}^{\prime}(0)=0 .
\end{gathered}
$$

1.2. In diesem Abschnitt betrachten wir die Aufgabe (1.6) mit einer Randbedingung

$$
f_{-}^{\prime}(l) N+f(l)=0
$$

am rechten Endpunkt $l$, wobei $N$ eine reelle Konstante (einschließlich $\infty$ ) bezeichne ${ }^{1}$. Dieser Randwertaufgabe läßt sich eine bezüglich des Eigenwertparameters $\lambda$ quadratische Operatorenschar in $L^{2}{ }_{V}$ zuordnen.

Wir setzen

\footnotetext{
${ }^{1}$ Für $N=\infty$ interpretieren wir (1.7) als $f_{-}^{\prime}(l)=0$.
} 


$$
\begin{gathered}
\mathfrak{D}\left(A_{N}\right):=\left\{f \in \mathfrak{D}_{0}: f_{-}^{\prime}(0)=0, f_{-}^{\prime}(l) N+f(l)=0\right\}, \\
A_{N} f:=-\varphi,
\end{gathered}
$$

wenn $f$ die Darstellung (1.3) besitzt. Man sieht leicht, daß diese Definition korrekt ist, d.h., aus $f=0$ (in $L_{V}^{2}$ ) folgt $\varphi=0$ (in $L_{V}^{2}$ ). Weiter sei für $f \in L_{V}^{2}$ :

$$
\begin{aligned}
(B f)(x) & :=\beta(x) f(x), \\
(C f)(x) & :=\gamma(x) f(x)
\end{aligned}
$$

mit den Funktionen $\beta$ und $\gamma$ aus (1.1). Die Operatoren $B$ und $C$ sind beschränkt, selbstadjungiert und miteinander vertauschbar, $C$ ist nichtnegativ. Außerdem gilt auf Grund von (1.2)

$$
|B|+C=I .
$$

Die Randwertaufgabe (1.6), (1.7) ist dann äquivalent mit der Gleichung

$$
-A_{N} f+\lambda B f+\lambda^{2} C f=g
$$

in $L_{V}^{2}$. Gilt $0 \leqq N<\infty$ (bzw. $N=\infty$ ), so ist der Operator $A_{N}$ streng positiv (bzw. nichtnegativ und der Nullpunkt ist Eigenwert von $A_{\infty}$ ).

Wir setzen zunächst $0 \leqq N<\infty$ voraus. Von der in (1.9) auftretenden Schar

$$
L_{N}(\lambda):=-A_{N}+\lambda B+\lambda^{2} C
$$

gehen wir über zur Schar

$$
\tilde{L}_{N}(\mu):=\mu^{2} I-\mu B_{1}-C_{1}
$$

mit $B_{1}=A_{N}^{-1 / 2} B A_{N}^{-1 / 2}, C_{1}=A_{N}^{-1 / 2} C A_{N}^{-1 / 2}$. Ein Punkt $\lambda_{0} \neq 0$ gehört offensichtlich genau dann zum Spektrum der Schar $L_{N}$ (d.h. $L_{N}\left(\lambda_{0}\right)$ besitzt keine auf ganz $L_{V}^{2}$ definierte Inverse), wenn $\mu_{0}:=1 / \lambda_{0}$ zum Spektrum der Schar $\tilde{L}_{N}$ gehört. Der Operator $A_{N}^{-1}$ und damit auch die Operatoren $B_{1}$ und $C_{1}$ sind kompakt in $L_{V}^{2}$, deshalb ist das Spektrum von $\tilde{L}_{N}$ außerhalb des Nullpunktes diskret; da $C_{1} \geqq 0$ gilt, ist es sogar reell. Wir bezeichnen die Anzahl der positiven (bzw. negativen) Eigenwerte von $\tilde{L}_{N}$ mit $n_{+}$(bzw. $\left.n_{-}\right)\left(0 \leqq n_{ \pm} \leqq \infty\right)$ und diese Eigenwerte selbst mit

$$
\mu_{+}^{(1)} \geqq \mu_{+}^{(2)} \geqq \cdots \quad\left(\text { bzw. } \mu_{-}^{(1)} \leqq \mu_{-}^{(2)} \leqq \cdots\right) ;
$$

dabei werde jeder Eigenwert so oft gezählt, wie seine Vielfachheit angibt ${ }^{1}$.

Für $u \in L_{V}^{2}, \quad\|u\|=1$, hat die quadratische Gleichung

\footnotetext{
1 Später werden wir sehen, daß diese Eigenwerte alle einfach sind.
} 


$$
\left(\tilde{L}_{N}(\mu) u, u\right) \equiv \mu^{2}-\mu\left(B_{1} u, u\right)-\left(C_{1} u, u\right)=0
$$

zwei reelle Lösungen $\mu_{ \pm}(u)$ :

$$
\mu_{ \pm}(u)=\frac{\left(B_{1} u, u\right)}{2} \pm \sqrt{\frac{\left(B_{1} u, u\right)^{2}}{4}+\left(C_{1} u, u\right)} .
$$

Die positiven (bzw. negativen) Eigenwerte der Schar $\tilde{L}_{N}$ lassen sich dann bekanntlich als Minmaxwerte des Funktionals $\mu_{+}$(bzw. $\left.\mu_{-}\right)$charakterisieren $([7],[17])$ :

$$
\begin{aligned}
& \mu_{+}^{(j)}=\max \min \mu_{+}(u), \quad j=1,2, \ldots, n_{+},
\end{aligned}
$$

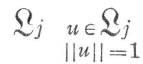

$$
\begin{aligned}
& \mu_{-}^{(\jmath)}=\min \max \mu_{-}(u), \quad j=1,2, \ldots, n_{-} \text {; } \\
& \Omega_{j} \quad u \in \Omega_{j}
\end{aligned}
$$

dabei bezeichnet $\mathfrak{a}_{j}$ einen Teilraum der Dimension $j$.

Im folgenden sei $x_{+}^{\prime \prime}$ (bzw. $\left.x_{-}^{\prime \prime}\right)$ die Anzahl der Wachstumspunkte der Funktion $M_{+}+D$ (bzw. $\left.M_{-}+D\right)$.

$\mathrm{S}$ a tz 1.1. Es gilt $n_{ \pm}=x_{ \pm}^{\prime \prime}$.

Beweis. Wir beweisen nur die Beziehung für die positiven Eigenwerte. Ist $\varkappa_{+}^{\prime \prime}$ endlich, so bezeichne $\mathfrak{\Omega}$ denjenigen Teilraum von $L_{V}^{2}$, dessen Elemente höchstens auf den Sprungpunkten von $M_{+}+D$ verschieden von Null sind. Dann gilt $\operatorname{dim} \mathfrak{L}=\varkappa_{+}^{\prime \prime}$ und

$$
\frac{(B u, u)}{2}+\sqrt{\frac{(B u, u)^{2}}{4}+(C u, u)}>0
$$

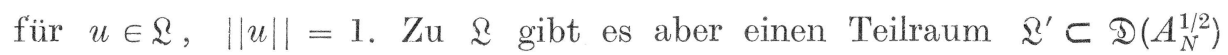
derselben Dimension $x_{+}^{\prime \prime}$, für dessen Elemente $u$ mit $\|u\|=1$ die Beziehung (1.12) besteht. Das Funktional $\mu_{+}$ist also auf dem $x_{+}^{\prime \prime}$-dimensionalen Teilraum $A_{N}^{1 / 2} \mathcal{Q}^{\prime}$ positiv, also gilt wegen (1.11) $n_{+} \geqq x_{+}^{\prime \prime}$. $\hat{\Omega}$ mit

Wäre $n_{+}>x_{+}^{\prime \prime}$, dann gäbe es einen $\left(x_{+}^{\prime \prime}+1\right)$-dimensionalen Teilraum

$$
\mu_{+}(u)>0 \quad \text { für } u \in \hat{\mathfrak{Q}},\|u\|=1,
$$

d. h., auch die Beziehung (1.12) würde für alle $u$ mit $\|u\|=1$ aus einem $\left(x_{+}^{\prime \prime}+1\right)$-dimensionalen Teilraum $\tilde{\mathfrak{L}}=A_{N}^{-1 / 2} \hat{\mathfrak{Q}}$ bestehen. Die Funktion $D$ habe genau $m_{+}$Wachstumspunkte $\left(m_{+} \leqq x_{+}^{\prime \prime}\right)$, d.h., der Wertebereich von $C$ ist $m_{+}$-dimensional. Dann gilt $(C u, u)=0$ für alle $u$ aus einem mindestens $\left(\varkappa_{+}^{\prime \prime}+1-m_{+}\right)$-dimensionalen Teilraum $\tilde{\Omega}^{\prime}$ von $\tilde{\mathbb{Q}}$, also ist

$$
(B u, u)>0 \quad \text { für alle } u \in \tilde{\Omega}^{\prime},\|u\|=1 .
$$


Da die Operatoren $B$ und $C$ vertauschbar sind, hat somit $B$ mindestens $x_{+}^{\prime \prime}+1-m_{+}$positive Eigenwerte, deren Eigenelemente im Orthogonalkomplement des Wertebereiches von $C$ liegen. Dann hat aber die Funktion $M_{+}$mindestens so viele Wachstumspunkte, die nicht Sprungpunkte von $D$ sind, d. h. $M_{+}+D$ hat mindestens $x_{+}^{\prime \prime}+1$ Wachstumspunkte, Widerspruch.

Ist $x_{+}^{\prime \prime}=\infty$, so ergibt sich die Behauptung ebenfalls leicht aus dem ersten Teil des Beweises.

Da nach Voraussetzung die Funktion $V$ unendlich viele Wachstumspunkte hat, gilt die

Folgerung 1.2. Die Schar $L_{N}$ hat für $0 \leqq N<\infty$ unendlich viele Eigenwerte; diese sind alle reell.

Aus den Betrachtungen in Abschnitt 1.4 wird sich ergeben, daß die erste Aussage der Folgerung 1.2 sogar für alle reellen $N$ (einschließlich $\infty$ ) richtig ist; dabei sind im Falle $0 \leqq N \leqq \infty$ alle Eigenwerte der Schar $L_{N}$ reell, im Falle $-\infty<N<0$ kann außerhalb der reellen Achse höchstens ein zur reellen Achse symmetrisch gelegenes Paar von Eigenwerten auftreten.

1.3. Im folgenden spielen die Lösungen $\varphi(\cdot ; \lambda)$ und $\psi(\cdot ; \lambda)$ der Differentialgleichung (1.4) mit $g=0$ und den Anfangsbedingungen

$$
\begin{array}{ll}
\varphi(0 ; \lambda)=1, & \varphi_{-}^{\prime}(0 ; \lambda)=0, \\
\psi(0 ; \lambda)=0, & \psi_{-}^{\prime}(0 ; \lambda)=1,
\end{array}
$$

d. h. die Lösungen der Integralgleichungen

$$
\begin{aligned}
& \varphi(x ; \lambda)=1-\int_{0}^{x}(x-s) \varphi(s ; \lambda) d V_{\lambda}(s), \\
& \psi(x ; \lambda)=x-\int_{0}^{x}(x-s) \psi(s ; \lambda) d V_{\lambda}(s)
\end{aligned}
$$

eine wesentliche Rolle; dabei haben wir

$$
d V_{\lambda}(s):=\lambda d M(s)+\lambda^{2} d D(s)
$$

gesetzt. Löst man z. B. die Gleichung (1.13) durch Iteration

$$
\begin{aligned}
& \varphi_{0}(x ; \lambda): \equiv 1 ; \\
& \varphi_{m}(x ; \lambda):=1-\int_{0}^{x}(x-s) \varphi_{m-1}(s ; \lambda) d V_{\lambda}(s), \quad m=1,2, \ldots,
\end{aligned}
$$


so ergibt sich

$$
\begin{array}{r}
(1.15) \varphi(x ; \lambda)=1-\int_{0}^{x-0}(x-s) d V_{\lambda}(s)+\int_{0}^{x-0} \int_{0}^{s_{2}-0}\left(x-s_{2}\right)\left(x-s_{1}\right) d V_{\lambda}\left(s_{1}\right) d V_{\lambda}\left(s_{2}\right) \\
-\ldots+(-1)^{m} \int_{0}^{x} \int_{0}^{s_{m}-0} \ldots \int_{0}^{s_{2}-0}\left(x-s_{m}\right)\left(s_{m}-s_{m-1}\right) \ldots\left(s_{2}-s_{1}\right) d V_{\lambda}\left(s_{1}\right) \ldots d V_{\lambda}\left(s_{m}\right) \\
+\ldots .
\end{array}
$$

Gemäß der Abschätzung aus [3], § 2.3, gilt für das allgemeine Glied der Reihe (1.15)

$$
\begin{aligned}
& \left|\int_{0}^{x} \int_{0}^{x} \ldots \int_{0}^{s_{m}-0}\left(x-s_{m}\right)\left(s_{m}-s_{m-1}\right) \ldots\left(s_{2}-s_{1}\right) d V_{\lambda}\left(s_{1}\right) \ldots d V_{\lambda}\left(s_{m}\right)\right| \\
& \leqq
\end{aligned}
$$

Deshalb konvergiert diese Reihe in jedem kompakten Teil der $\lambda$-Ebene gleichmäßig und definiert eine ganze Funktion $\varphi(x ; \cdot)$ der Ordnung höchstens gleich eins, genauer, es gilt

$$
|\varphi(x ; \lambda)| \leqq \cosh \left(2 x\left(|\lambda||M|(x-0)+|\lambda|^{2} D(x-0)\right)\right)^{1 / 2} .
$$

Die Funktion $\varphi(\cdot ; \lambda)$ ist absolutstetig in $[0, l]$; sie hat in jedem Punkt $x$ des Intervalls $[0, l)$ (bzw. $(0, l])$ eine rechts- (bzw. links-) seitige Ableitung

$$
\varphi_{ \pm}^{\prime}(x ; \lambda)=-\int_{0}^{x \pm 0} \varphi(s ; \lambda) d V_{\lambda}(s),
$$

die bezüglich $\lambda$ ebenfalls ganze Funktionen der Ordnung höchstens gleich eins sind.

In gleicher Weise zeigt man die Existenz einer Lösung $\psi$ der Integralgleichung (1.14), die (ebenso wie ihre rechts- und linksseitigen Ableitungen $\psi_{ \pm}^{\prime}$ ) bezüglich $\lambda$ eine ganze Funktion der Ordnung höchstens gleich eins ist. Schließlich vermerken wir noch die für beliebige komplexe $\lambda$ und $x \in[0, l]$ bestehende Identität

$$
\varphi(x ; \lambda) \psi_{+}^{\prime}(x ; \lambda)-\varphi_{+}^{\prime}(x ; \lambda) \psi(x ; \lambda) \equiv 1
$$

sowie die Beziehungen

$$
\varphi(x ; \bar{\lambda})=\overline{\varphi(x ; \lambda)}, \quad \psi(x ; \bar{\lambda})=\overline{\psi(x ; \lambda)} .
$$


Wir betrachten die Randwertaufgabe

$$
\begin{gathered}
d f_{+}^{\prime}(x)+\lambda f(x) d M(x)+\lambda^{2} f(x) d D(x)=0, \quad 0 \leqq x \leqq l, \\
f_{-}^{\prime}(0)=0, \quad f(l)=0 .
\end{gathered}
$$

Sie hat offensichtlich genau für diejenigen Werte von $\lambda$ eine nichttriviale Lösung, für die $\varphi(l ; \lambda)=0$ gilt, und diese (bis auf einen konstanten Faktor eindeutige) Lösung ist die Funktion $\varphi(\cdot ; \lambda)$. Da andererseits diese Punkte $\lambda$ die Eigenwerte der Schar $L_{0}$ (siehe (1.10)) sind, ergibt sich aus der Folgerung 1.2 das

L e m m a 1.3. Die Funktion $\varphi(l ; \cdot)$ hat unendlich viele reelle Nullstellen.

Mit $N_{\varkappa}$ bezeichnen wir im folgenden die Menge derjenigen Funktionen $N$, die in der oberen Halbebene meromorph sind und für die der Kern $K_{N}$

$$
K_{N}(z, \zeta)=\frac{N(z)-\overline{N(\zeta)}}{z-\bar{\zeta}} \quad\left(z, \zeta \in Z_{N}\right)
$$

auf dem Holomorphiegebiet $Z_{N}$ von $N$ genau $x$ negative Quadrate hat (d. h., jede der Formen $\sum_{i, j=1}^{n} K_{N}\left(z_{i}, z_{j}\right) \zeta_{i} \bar{\zeta}_{j}, z_{i} \in Z_{N}$, hat höchstens $x$ und mindestens eine dieser Formen hat genau $x$ negative Quadrate). Die Funktionen $N \in N_{\varkappa}$ setzen wir durch Spiegelung $N(z)=\overline{N(z)}$ auf die untere Halbebene fort; außerdem sei

$$
N_{\varkappa}:= \begin{cases}N_{0} \cup\{\infty\}, & x=0, \\ N_{\varkappa}, & x \geqq 1 .\end{cases}
$$

Die Klasse $N_{0}$ stimmt bekanntlich mit der von R. Nevanlinna eingeführten Klasse der in der oberen Halbebene definierten und holomorphen Funktionen $N$ mit $\operatorname{Im} N(z) / \operatorname{Im} z \geqq 0$ überein; die Klassen $N_{\varkappa}$ wurden in [6] eingeführt und untersucht.

Schließlich bezeichnen wir für eine auf einem Gebiet $G$ der komplexen Ebene erklärte Funktion $F$ mit $\hat{F}$ die durch Multiplikation mit der unabhängigen Veränderlichen entstehende Funktion: $\hat{F}(\lambda):=\lambda F(\lambda)$ $(\lambda \in G)$.

L e $\mathrm{m} \mathrm{m}$ a 1.4. Es gilt

$$
\frac{\widehat{\varphi(l ; \cdot)}}{\varphi_{-}^{\prime}(l ; \cdot)} \in N_{0} .
$$

Beweis. Wir gehen aus von der Gleichung 


$$
\lambda \int_{0}^{l} \varphi(x ; \lambda) \overline{\varphi(x ; \mu)} d M(x)+\lambda^{2} \int_{0}^{l} \varphi(x ; \lambda) \overline{\varphi(x ; \mu)} d D(x)
$$

$=-\int_{0}^{l} d \varphi_{+}^{\prime}(x ; \lambda) \overline{\varphi(x ; \mu)}=-\varphi_{-}^{\prime}(l ; \lambda) \overline{\varphi(l ; \mu)}+\int_{0}^{l} \varphi_{+}^{\prime}(x ; \lambda) \overline{\varphi_{+}^{\prime}(x ; \mu)} d x$.

Multiplizieren wir sie mit $\bar{\mu}$, schreiben die durch Vertauschung von $\lambda$ und $\bar{\mu}$ entstehende Beziehung auf und subtrahieren beide voneinander, so ergibt sich

$$
\begin{aligned}
& \left.\lambda \overline{\varphi_{-}^{\prime}(l ; \mu}\right) \varphi(l ; \lambda)-\bar{\mu} \varphi_{-}^{\prime}(l ; \lambda) \overline{\varphi(l ; \mu)} \\
& =(\lambda-\bar{\mu})\left\{\lambda \bar{\mu} \int_{0}^{l} \varphi(x ; \lambda) \overline{\varphi(x ; \mu)} d D(x)+\int_{0}^{l} \varphi_{+}^{\prime}(x ; \lambda) \overline{\varphi_{+}^{\prime}(x ; \mu)} d x\right\}
\end{aligned}
$$

und daraus leicht die Behauptung.

Folgerung 1.5. Die Funktion $\varphi_{-}^{\prime}(l ; \cdot)$ hat unendlich viele Nullstellen. Alle Nullstellen von $\varphi(l ; \cdot)$ und $\varphi_{-}^{\prime}(l ; \cdot)$ sind reell.

Aus der Beziehung (1.17) erhält man durch ähnliche einfache Umformungen die Gleichung

$$
\begin{aligned}
& \frac{p(l ; \lambda)}{\varphi_{-}^{\prime}(l ; \lambda)}-\frac{\overline{\varphi(l ; \mu)}}{\overline{\varphi_{-}^{\prime}(l ; \mu)}} \\
& =(\lambda-\bar{\mu}) \frac{3}{\varphi_{-}^{\prime}(l ; \lambda) \overline{\varphi_{-}^{\prime}(l ; \mu)}}\left[\int_{0}^{l} \varphi(x ; \lambda) \overline{\varphi(x ; \mu)}(d M(x)+(\lambda+\bar{\mu}) d D(x))\right],
\end{aligned}
$$

und daraus das

$\mathrm{L}$ e $\mathrm{m} \mathrm{m}$ a 1.6. Hat die (nichtabnehmende) Funktion $D+M_{-}$nur endlich viele (undzwar $x^{\prime \prime}$ ) Wachstumspunkte, so gilt für ein $x$ mit $0 \leqq x \leqq x^{\prime \prime}$

$$
\frac{\varphi(l ; \cdot)}{\varphi_{-}^{\prime}(l ; \cdot)} \in N_{\varkappa} .
$$

1.4. Die im folgenden auftretenden Funktionen $N$ seien stückweise meromorph oder identisch gleich $\infty$ in einem Gebiet $G_{N}$, das die untere und obere Halbebene enthält, und es gelte $N(\bar{\lambda})=\overline{N(\lambda)}$. Mit den Funktionen $\varphi$ und $\psi$ aus Abschnitt 1.3 setzen wir

$$
m_{N}(\lambda):=\frac{\psi_{-}^{\prime}(l ; \lambda) N(\lambda)+\psi(l ; \lambda)}{\varphi_{-}^{\prime}(l ; \lambda) N(\lambda)+\varphi(l ; \lambda)} \quad\left(\lambda \in G_{N}\right) .
$$


Da auf Grund der Identität (1.16) Zähler und Nenner nicht gleichzeitig verschwinden, ist $m_{N}(\lambda)$ für $\lambda \in \boldsymbol{G}_{N}$ definiert (eventuell $=\infty$ ), und es gilt $m_{N}(\bar{\lambda})=\overline{m_{N}(\lambda)}$. Der Quotient $m_{N}$ ist der Weylsche Koeffizient der Aufgabe

$$
\begin{gathered}
d f_{+}^{\prime}(x)+\lambda f(x) d M(x)+\lambda^{2} f(x) d D(x)=0,0 \leqq x \leqq l \\
f_{-}^{\prime}(0)=0
\end{gathered}
$$

der zur Randbedingung

$$
f_{-}^{\prime}(l) N(\lambda)+f(l)=0
$$

gehört, d. h., die Funktion $\chi(\cdot ; \lambda)$ :

$$
\begin{array}{ll}
\chi(x ; \lambda):=-\psi(x ; \lambda)+m_{N}(\lambda) \varphi(x ; \lambda) & \left(m_{N}(\lambda) \neq \infty\right) \\
\chi(x ; \lambda):=\varphi(x ; \lambda) & \left(m_{N}(\lambda)=\infty\right)
\end{array}
$$

genügt der Differentialgleichung aus (1.18) und der Randbedingung (1.19). Die Punkte $\lambda$ mit $m_{N}(\lambda)=\infty$ sind insbesondere genau diejenigen Punkte aus $G_{N}$, für welche die Randwertaufgabe (1.18), (1.19) eine nichttriviale Lösung hat. Wir nennen solche Punkte $\lambda$ im folgenden wieder Eigenwerte der Randwertaufgabe (1.18), (1.19) und die zugehörigen Lösungen Eigenfunktionen. Offensichtlich ist die Funktion $\varphi(\cdot ; \lambda)$ die (bis auf einen konstanten Faktor) einzige Eigenfunktion zum Eigenwert $\lambda \in G_{N}$ der Randwertaufgabe (1.18), (1.19).

Satz 1.7. (a) Gilt $N \in \tilde{N}_{x^{\prime}}$, und hat die (nichtabnehmende) Funktion $D+M_{-}$nur endlich viele (und zwar $x^{\prime \prime}$ ) Wachstumspunkte, so gehört der Weylsche Koeffizient $m_{N}$ zu einer Klasse $N_{x}$ mit $0 \leqq x \leqq x^{\prime}+x^{\prime \prime}$.

(b) Gilt $\hat{N} \in \tilde{N}_{x^{\prime}}$, so gehört die Funktion $\hat{m}_{N}$ zu einer Klasse $N_{\varkappa}$ mit $0 \leqq x \leqq x^{\prime}$

Beweis. Wir überlegen uns zunächst, daß unter den Voraussetzungen von (a) oder (b) die Funktion $m_{N}$ nicht identisch gleich $\infty$ ist. Anderenfalls wäre nämlich

$$
N(\lambda)=-\frac{\varphi(l ; \lambda)}{\varphi_{-}^{\prime}(l ; \lambda)} \quad \text { für alle } \lambda \neq \bar{\lambda},
$$

also hätte im Falle (a) der Kern $K_{N}$ in der oberen Halbebene endlich viele positive Quadrate (Lemma 1.6). Da er nach Voraussetzung auch nur endlich viele negative Quadrate hat, muß $N$ nach den Ergebnissen von [6] eine rationale Funktion sein. Das widerspricht den Aussagen von Lemma 1.3 und Folgerung 1.5. Im Falle (b) folgt aus (1.21) und Lemma 1.4, daß der Kern $K_{N}$ negativ definit ist, nach Voraussetzung hat er aber nur endlich viele negative Quadrate. Damit ergibt sich wie im Falle (a) ein Widerspruch, 
Wir haben nur Punkte $\lambda$ mit $m_{N}(\lambda) \neq \infty$ zu betrachten. Die Funktion $\chi(\cdot ; \lambda)$ aus $(1.20)$ genügt den Randbedingungen

$$
\begin{gathered}
\chi(0 ; \lambda)=m_{N}(\lambda), \quad \chi_{-}^{\prime}(0 ; \lambda)=-1, \\
\chi_{-}^{\prime}(l ; \lambda) N(\lambda)+\chi(l ; \lambda)=0 .
\end{gathered}
$$

Deshalb ergibt sich (vgl. (1.17)) einerseits durch partielle Integration

$$
\begin{aligned}
& \int_{0}^{l} d \chi_{+}^{\prime}(x ; \lambda) \overline{\chi(x ; \mu)}=\left(\left.\chi_{+}^{\prime}(x ; \lambda) \overline{\chi(x ; \mu))}\right|_{0} ^{l}-\int_{0}^{l} \chi_{+}^{\prime}(x ; \lambda) \overline{\chi_{+}^{\prime}(x ; \mu)} d x\right. \\
& \left.=-\overline{N(\mu)} \chi_{-}^{\prime}(l ; \lambda) \overline{\chi_{-}^{\prime}(l ; \mu}\right)+\overline{m_{N}(\mu)}-\int_{0}^{l} \chi_{+}^{\prime}(x ; \lambda) \overline{\chi_{+}^{\prime}(x ; \mu)} d x,
\end{aligned}
$$

andererseits folgt bei Beachtung der Differentialgleichung

$$
\begin{aligned}
& \int_{0}^{l} d \chi_{+}^{\prime}(x ; \lambda) \overline{\chi(x ; \mu)} \\
& =-\lambda \int_{0}^{l} \chi(x ; \lambda) \overline{\chi(x ; \mu)} d M(x)-\lambda^{2} \int_{0} \chi(x ; \lambda) \overline{\chi(x ; \mu)} d D(x),
\end{aligned}
$$

d. h., es gilt

$$
\begin{aligned}
& \overline{m_{N}(\mu)}=\overline{N(\mu)} \chi_{-}^{\prime}(l ; \lambda) \overline{\chi_{-}^{\prime}(l ; \mu)}+\int_{0}^{l} \chi_{+}^{\prime}(x ; \lambda) \overline{\chi_{+}^{\prime}(x ; \mu)} d x \\
& -\lambda \int_{0}^{l} \chi(x ; \lambda) \overline{\chi(x ; \mu)} d M(x)-\lambda^{2} \int_{0}^{l} \chi(x ; \lambda) \overline{\chi(x ; \mu)} d D(x) .
\end{aligned}
$$

Schreiben wir noch die durch Vertauschung von $\lambda$ und $\bar{\mu}$ entstehende Beziehung auf und subtrahieren von ihr (1.22), so erhalten wir nach Division durch $\lambda-\bar{\mu}$

$$
\begin{aligned}
& \frac{m_{N}(\lambda)-\overline{m_{N}(\mu)}}{\lambda-\bar{\mu}}=\chi_{-}^{\prime}(l ; \lambda) \overline{\chi_{-}^{\prime}(l ; \mu)} \frac{N(\lambda)-\overline{N(\mu)}}{\lambda-\bar{\mu}}+\int_{0}^{l} \chi_{+}^{\prime}(x ; \lambda) \overline{\chi(x ; \mu)} d M(x) \\
& +\lambda \bar{\mu} \int_{0}^{l} \chi(x ; \lambda) \overline{\chi(x ; \mu)} d D(x),
\end{aligned}
$$

woraus leicht die Aussage (a) folgt. 
Nach Multiplikation von (1.22) mit $\bar{\mu}$ ergibt sich auf gleiche Weise

$$
\begin{aligned}
& \frac{\lambda m_{N}(\lambda)-\bar{\mu} \overline{m_{N}(\mu)}}{\lambda-\bar{\mu}}=\frac{\overline{N(\lambda)}-\bar{\mu} \overline{N(\mu)}}{\lambda-\bar{\mu}} \chi^{\prime}(l ; \lambda) \overline{\chi^{\prime}(l ; \mu)} \\
& +\int_{0}^{l} \chi_{+}^{\prime}(x ; \lambda) \overline{\chi_{+}^{\prime}(x ; \mu)} d x+\lambda \bar{\mu} \int_{0}^{l} \chi(x ; \lambda) \overline{\chi(x ; \mu)} d D(x)
\end{aligned}
$$

and daraus die Aussage (b) des Satzes.

Eine Funktion der Klasse $N_{\varkappa}$ hat nach den Ergebnissen von [6] höchstens $\varkappa$ Pole in der oberen (oder unteren) Halbebene (jeder so oft gezählt, wie seine Vielfachheit angibt). Da andererseits die Pole von $m_{N}$ genau die Eigenwerte der Randwertaufgabe (1.18), (1.19) im Analytizitätsgebiet von $m_{N^{\prime}}$ sind, ergibt sich die

Folger ung 1.8. Unter den Voraussetzungen und Bezeichnungen von (a) oder (b) aus Satz 1.7 hat die Randwertaufgabe (1.18), (1.19) höchstens * Paare nichtreeller Eigenwerte; jedes solche Paar liegt symmetrisch zur reellen Achse.

F o lg er u ng 1.9. Jede der Funktionen $\psi(l ; \cdot), \psi_{-}^{\prime}(l ; \cdot)$ hat unendlich viele Nullstellen; diese sind alle reell.

Gemäß Satz 1.7 (b), gehören nämlich die Funktionen

$$
\hat{m}_{0}: \hat{m}_{0}(\lambda)=\lambda \frac{\psi(l ; \lambda)}{\varphi(l ; \lambda)}
$$

und

$$
\hat{m}_{\infty}: \quad \hat{m}_{\infty}(\lambda)=\lambda \frac{\psi_{-}^{\prime}(l ; \lambda)}{\varphi_{-}^{\prime}(l ; \lambda)}
$$

zur Klasse $N_{0}$. Da sie nach Lemma 1.3 und Folgerung 1.5 unendlich viele Pole haben, folgt die Aussage aus bekannten Eigenschaften von Funktionen der Klasse $N_{0}$.

Wir bemerken, daß sich aus Lemma 1.4 und Lemma 1.6 sowie aus Satz 1.7 z. B. auch Trennungseigenschaften der Nullstellen von $\varphi\left(l ;{ }^{\bullet}\right)$, $\varphi_{-}^{\prime}(l ; \cdot), \psi(l ; \cdot), \psi_{-}^{\prime}(l ; \cdot)$ ergeben. Außerdem kann man im Falle $N(\lambda)$ $\equiv c(=\bar{c})$ Aussagen über die Abhängigkeit der Eigenwerte der Randwertaufgabe (1.18), (1.19) von $c$ erhalten (vgl. [8], § 3.4).

Unter der Voraussetzung $\hat{N} \in \tilde{N}_{0}$ gehört die Funktion $\hat{m}_{N}$ nach Satz 1.7 (b) zur Klasse $N_{0}$. Im nächsten Abschnitt führen wir für die Anfangswertaufgabe (1.18) eine Klasse von Spektralfunktionen ein, die gerade den Randbedingungen (1.19) mit $\hat{N} \in \tilde{N}_{0}$ entsprechen und sehr eng mit $\hat{m}_{N}$ zusammenhängen. Ist die Bedingung $\hat{N} \in \tilde{N}_{0}$ nicht erfüllt, gilt aber noch $\hat{N} \in \tilde{N}_{\varkappa^{\prime}}$ mit einem $\varkappa^{\prime}>0 \quad$ (z. B. im Falle $N(\lambda) \equiv c<0$, dann ist $\varkappa^{\prime}=1$ ), 
so läßt sich mit Hilfe der Funktion $\hat{m}_{N}\left(\in N_{\varkappa}\right)$ ebenfalls eine Spektralfunktion einführen. Thre Definition wird jedoch durch das Auftreten sog. kritischer Punkte komplizierter (vgl. [6], in abstrakter Darstellung auch [5]).

1.5. Für beschränkte meßbare Funktionen $f$ auf $[0, l]$ definieren wir zwei Fouriertransformationen

$$
\begin{aligned}
& f \rightarrow F_{1}^{[f]}: \quad F_{1}^{[f]}(\lambda)= \begin{cases}\frac{1}{\lambda} \int_{0}^{l} f(x) \varphi_{+}^{\prime}(x ; \lambda) d x & (\lambda \neq 0) ; \\
-\int_{0}^{l} f(x) M(x) d x & (\lambda=0)\end{cases} \\
& \begin{array}{l}
l \\
f \rightarrow F_{2}^{[f]}:
\end{array} F_{2}^{[f]}(\lambda)=\int_{0}^{l} f(x) \varphi(x ; \lambda) d D(x) .
\end{aligned}
$$

Ist $u \in \mathfrak{D}_{0}, u(l)=0$, so folgt durch partielle Integration

$$
F_{1}^{\left[u_{+}^{\prime}{ }^{]}\right.}(\lambda)=\int_{0}^{l} u(x) \varphi(x ; \lambda)(d M(x)+\lambda d D(x)) .
$$

Definition 1.10. Eine Funktion $\tau$, definiert auf $(-\infty, \infty)$, heiße Spektralfunktion der Anfangswertaufgabe (1.18), wenn sie die folgenden Eigenschaften hat:

(i) $\tau$ ist nichtfallend, $\quad \tau(0)=0, \quad \tau(\lambda)=\frac{1}{2}(\tau(\lambda+0)+\tau(\lambda-0))$ $(-\infty<\lambda<\infty)$;

(ii) für beschränkte meßbare Funktionen $f$ auf $[0, l]$ gilt

$$
\begin{aligned}
& \int_{0}^{l}|f(x)|^{2} d x=\int_{-\infty}^{\infty}\left|F_{1}^{[f]}(\lambda)\right|^{2} d \tau(\lambda), \\
& \int_{0}^{l}|f(x)|^{2} d D(x)=\int_{-\infty}^{\infty}\left|F_{2}^{[f]}(\lambda)\right|^{2} d \tau(\lambda), \\
& \int_{-\infty}^{\infty} F_{1}^{[f]}(\lambda) \overline{F_{2}^{[f]}(\lambda)} d \tau(\lambda)=0 .
\end{aligned}
$$

Die Spektralfunktion $\tau$ heißt orthogonal, wenn die Menge aller Fouriertransformierten $F_{1}^{[f]}, F_{2}^{[f]}$ der beschränkten meßbaren Funktionen $f$ auf $[0, l]$ in $L_{\tau}^{2}$ dicht liegt. 
Die Bedingung (ii) ist offensichtlich äquivalent der folgenden:

Für beliebige beschränkte meßbare Funktionen $f, g$ auf $[0, l]$ gilt

$$
\begin{gathered}
\int_{0}^{l} f(x) \overline{g(x)} d x=\int_{-\infty}^{\infty} F_{1}^{[f]}(\lambda) \overline{F_{1}^{[g]}(\lambda)} d \tau(\lambda), \\
\int_{0}^{l} f(x) \overline{g(x)} d D(x)=\int_{-\infty}^{\infty} F_{2}^{[f]}(\lambda) \overline{F_{2}^{[g]}(\lambda)} d \tau(\lambda), \\
\int_{-\infty}^{\infty} F_{1}^{[f]}(\lambda) \overline{F_{2}^{[g]}(\lambda)} d \tau(\lambda)=0 .
\end{gathered}
$$

Wir überlegen uns, daß diese Definition der Spektralfunktion im Falle $D \equiv 0$ in die Definition der $A$-Spektralfunktion aus [8], $\S 3.1$, übergeht. Dabei war eine $A$-Spektralfunktion $\tau$ durch die Eigenschaft (i) aus Definition 1.10 und die Beziehung

$$
\int_{0}^{l}\left|f_{+}^{\prime}(x)\right|^{2} d x=\int_{-\infty}^{\infty}\left|F_{1}^{\left[f_{+}^{\prime}\right]}(\lambda)\right|^{2} d \tau(\lambda)
$$

für alle $f$ der Gestalt

$$
f(x)=f_{0}-\int_{0}^{x}(x-s) \varphi(s) d M(s) \quad \text { mit } \quad \varphi \in L_{|M|}^{2}, \quad f(l)=f_{-}^{\prime}(l)=0,
$$

definiert.

Ist $\tau$ eine Spektralfunktion (im Sinne von Definition 1.10), so besteht offensichtlich (1.27), d.h., $\tau$ ist eine $A$-Spektralfunktion. Für eine $A$ Spektralfunktion $\tau$ läßt sich andererseits die Beziehung (1.27) auf die abgeschlossene lineare Hülle in $L^{2}(0, l)$ der in (1.27) auftretenden Funktionen $f_{+}^{\prime}$ erweitern. Diese abgeschlossene lineare Hülle stimmt aber mit $L^{2}(0, l)$ überein, denn für $g_{0} \in L^{2}(0, l)$,

$$
0=\int_{0}^{l}\left(\int_{0}^{x+0} \varphi(s) d M(s)\right) \overline{g_{0}(x)} d x=\int_{0}^{l} \varphi(x) \int_{x}^{l} \overline{g_{0}(s)} d s d M(x)
$$

für alle $\varphi \in L_{|M|}^{2}$ mit $\int_{0}^{l} \varphi(x) d M(x)=0$ folgt $\int_{x}^{l} g_{0}(s) d s=c \quad(=$ const.), also $c=0$, und $g_{0}$ ist das Nullelement von $L^{2}(0, l)$. Damit ergibt sich insbesondere (1.24).

Es sei jetzt $\lambda \neq 0$. Dann läßt sich die Anfangswertaufgabe (1.18) 
folgendermaßen als Anfangswertaufgabe für ein kanonisches System umschreiben:

$$
\begin{gathered}
J d \boldsymbol{u}(x)=\lambda d \boldsymbol{H}(x) \boldsymbol{u}(x), \quad 0 \leqq x \leqq l, \\
\boldsymbol{u}(0)=\left(\begin{array}{l}
u_{0} \\
0
\end{array}\right) ;
\end{gathered}
$$

dabei ist

$$
\begin{gathered}
\boldsymbol{J}=\left(\begin{array}{rr}
0 & -1 \\
1 & 0
\end{array}\right), \quad \boldsymbol{u}(x)=\left(\begin{array}{l}
u(x) \\
v(x)
\end{array}\right), \\
H(x)=\left(\begin{array}{c}
D(x)+\int_{0}^{x} M^{2}(s) d s-\int_{0}^{x} M(s) d s \\
-\int_{0}^{x} M(s) d s
\end{array}\right),
\end{gathered}
$$

genauer, genügt die Funktion $u$ der Integralgleichung

$$
u(x)=u_{0}-\int_{0}^{x}(x-s) u(s)\left(\lambda d M(s)+\lambda^{2} d D(s)\right),
$$

so genügt die Vektorfunktion

$$
\boldsymbol{u}(x)=\left(\begin{array}{l}
u(x) \\
v(x)
\end{array}\right) \text { mit } v(x)=M(x) u(x)+\frac{1}{\lambda} u_{+}^{\prime}(x)
$$

der Integralgleichung:

$$
\boldsymbol{J}\left(\boldsymbol{u}(x)-\boldsymbol{u}_{0}\right)=\lambda \int_{0}^{x+0} d \boldsymbol{H}(s) \boldsymbol{u}(s), \quad \boldsymbol{u}_{0}=\left(\begin{array}{c}
u_{0} \\
0
\end{array}\right) .
$$

Das kanonische System in (1.28) ist von positivem Typ, d.h., es gilt $d \boldsymbol{H}(x) \geqq 0$ und $\int_{0}^{l} d \boldsymbol{H}(x)$ ist eine positiv definite Matrix. Die erste Beziehung ist offensichtlich. Falls für einen Vektor

$$
z:=\left(\begin{array}{c}
z_{1} \\
z_{2}
\end{array}\right)
$$

und für $z^{*}:=\left(\bar{z}_{1}, \bar{z}_{2}\right)$

$$
\int_{0}^{i} z^{*} d \boldsymbol{H}(x) z=0
$$


gilt, so folgt

$$
\int_{0}^{l} d D(x)\left|z_{1}\right|^{2}=0 \text { und } \int_{0}^{l}\left|M(x) z_{1}-z_{2}\right|^{2} d x=0 .
$$

Im Falle $D(l) \neq 0$ ergibt sich sofort $z_{1}=z_{2}=0$, im Falle $D(l)=0$ erhalten wir $M(x)=z_{2} / z_{1}$ für fast alle $x$ und daraus $M(x)=$ const. für $x \neq 0$, was der Voraussetzung widerspricht, daß $l$ ein Wachstumspunkt von $V$ sein sollte.

Ist allgemein ein kanonisches System

$$
\boldsymbol{J} d \boldsymbol{u}(x)=\lambda d \boldsymbol{H}(x) \boldsymbol{u}(x), \quad 0 \leqq x \leqq l,
$$

der Dimension zwei und von positivem Typ gegeben $\left(\boldsymbol{J}=-\boldsymbol{J}^{*}, \boldsymbol{J}^{2}\right.$ $\left.=-\boldsymbol{I}_{2}\right)^{1}$, so versteht man unter seinem Matrizanten $U(x ; \lambda)$ die (matrixwertige) Lösung der Anfangswertaufgabe

$$
\boldsymbol{J} d \boldsymbol{U}(x ; \lambda)=\lambda d \boldsymbol{H}(x) \boldsymbol{U}(x ; \lambda), \quad \boldsymbol{U}(0 ; \lambda)=\boldsymbol{I}_{2} .
$$

Es bezeichne $\boldsymbol{u}_{1}(x ; \lambda)$ die erste Spalte dieses Matrizanten und

$$
f=\left(\begin{array}{c}
f_{1} \\
f_{2}
\end{array}\right)
$$

eine beliebige Vektorfunktion mit beschränkten meßbaren Komponenten auf $[0, l]$. Dann definiert man bekanntlich (vgl. [3], § 14) die zur Anfangswertaufgabe

$$
\begin{gathered}
\boldsymbol{J} d \boldsymbol{u}(x)=\lambda d \boldsymbol{H}(x) \boldsymbol{u}(x), \quad 0 \leqq x \leqq l, \\
\boldsymbol{u}(0)=\left(\begin{array}{l}
1 \\
0
\end{array}\right),
\end{gathered}
$$

gehörige Fouriertransformation $F$ durch die Gleichung

$$
F^{[f]}(\lambda)=\int_{0}^{l}\left(f_{1}(x) f_{2}(x)\right) d \boldsymbol{H}(x) \boldsymbol{u}_{1}(x ; \lambda) .
$$

Eine Funktion $\tau$ mit der Eigenschaft (i) aus Definition 1.10 heißt Spektralfunktion der Aufgabe (1.31), wenn für jede Vektorfunktion $f$ mit den angegebenen Eigenschaften gilt:

$$
\left.\int_{-\infty}^{\infty} F^{[f]}(\lambda)\right|^{2} d \tau(\lambda)=\int_{0}^{l} f^{*}(x) d \boldsymbol{H}(x) \boldsymbol{f}(x) ;
$$

\footnotetext{
${ }^{1} \boldsymbol{I}_{2}$ bezeichnet die zweireihige Einheitsmatrix.
} 
die Spektralfunktion $\tau$ heißt orthogonal, wenn die Menge der Fourier transformierten aus (1.32) in $L_{\tau}^{2}$ dicht liegt ([3], § 14).

Haben für das kanonische System (1.30) $\boldsymbol{J}$ und $\boldsymbol{H}$ die in (1.29) angegebene spezielle Gestalt, so ergibt sich mit den Funktionen $\varphi$ und $\psi$ aus Abschnitt 1 für den Matrizanten leicht

$\boldsymbol{U}(x ; \lambda)=\left(\begin{array}{cc}p(x ; \lambda) & \lambda \psi(x ; \lambda) \\ \frac{1}{\lambda} \varphi_{+}^{\prime}(x ; \lambda)+M(x+0) \varphi(x ; \lambda) & \psi_{+}^{\prime}(x ; \lambda)+\lambda M(x+0) \psi(x ; \lambda)\end{array}\right)$.

L e m m a 1.11. Jede Spektralfunktion (bzw. orthogonale Spektralfunktion) der Anfangswertaufgabe (1.18) ist eine Spektralfunktion (bzw. orthogonale Spektralfunktion) der Anfangswertaufgabe (1.31), wenn $J$ und H durch (1.29) gegeben sind, und umgekehrt.

Dieses Lemma ergibt sich auf Grund der Beziehungen

$$
\begin{aligned}
& F^{[f]}(\lambda)=F_{1}^{[f]}(\lambda) \quad \text { für } f=\left(\begin{array}{c}
0 \\
f
\end{array}\right), \\
& F^{[g]}(\lambda)=F_{2}^{[g]}(\lambda) \quad \text { für } g=\left(\begin{array}{c}
g \\
M g
\end{array}\right) .
\end{aligned}
$$

Für $\hat{N} \in \tilde{N}_{0}$ gehört gemäß Satz 1.7 (b) die Funktion $\hat{m}_{N}$ :

$$
\hat{m}_{N}(\lambda)=\lambda \frac{\psi_{-}^{\prime}(l ; \lambda) N(\lambda)+\psi(l ; \lambda)}{p_{-}^{\prime}(l ; \lambda) N(\lambda)+\varphi(l ; \lambda)}
$$

zur Klasse $N_{0}$. Folglich definiert die Gleichung

$$
\tau_{N}(\lambda)=\frac{1}{\pi} \lim _{\varepsilon \downarrow 0} \int_{0}^{\lambda} \operatorname{Im} \hat{m}_{N}(\xi+i \varepsilon) d \xi
$$

eine Funktion $\tau=\tau_{N}$ mit den Eigenschaften (i) von Definition 1.10. Aus den allgemeinen Resultaten von I.S. Kac und M.G. Krein ([3], § 14) folgt jetzt leicht der

$\mathrm{Satz}$ 1.12. Die Gesamtheit der Spektralfunktionen der Anfangswertaufgabe (1.18) stimmt mit der Gesamtheit der Funktionen $\tau_{N^{*}}$ aus (1.34) überein, wenn $N$ die Menge aller Funktionen mit der Eigenschaft $\hat{N} \in \tilde{N}_{0}$ durchläuft. Dabei ergeben sich die orthogonalen Spektralfunktionen $\tau_{N^{*}}$ genau dann, wenn $\hat{N}$ eine reelle Konstante (einschließlich $\infty$ ) ist.

Beweis. Gemäß Lemma 1.11 genügt es, die Spektralfunktionen der Anfangswertaufgabe (1.28) mit $\boldsymbol{J}$ und $\boldsymbol{H}$ aus (1.29) zu beschreiben. Diese ergeben sich aber nach [3], § 14.4 und (1.33) gemäß (1.34) aus den zur Klasse $\boldsymbol{N}_{0}$ gehörenden Funktionen 


$$
\begin{aligned}
& \frac{\left(\psi_{-}^{\prime}(l ; \lambda)+\lambda M(l) \psi(l ; \lambda)\right) N_{1}(\lambda)+\lambda \psi(l ; \lambda)}{\left(\frac{1}{\lambda} \varphi_{-}^{\prime}(l ; \lambda)+M(l) \varphi(l ; \lambda)\right) N_{1}(\lambda)+\varphi(l ; \lambda)} \\
= & \lambda \frac{\psi_{-}^{\prime}(l ; \lambda) \frac{N_{1}(\lambda)}{\lambda\left(1+M(l) N_{1}(\lambda)\right)}+\psi(l ; \lambda)}{\varphi_{-}^{\prime}(l ; \lambda) \frac{N_{1}(\lambda)}{\lambda\left(1+M(l) N_{1}(\lambda)\right)}+\varphi(l ; \lambda)}=\hat{m}_{N}(\lambda)
\end{aligned}
$$

mit

$$
N(\lambda):=\frac{N_{1}(\lambda)}{\lambda\left(1+M(l) N_{1}(\lambda)\right)},
$$

wenn $N_{1}$ die Klasse $\tilde{N}_{0}$ durchläuft. Die Zuordnung $N_{1} \rightarrow N$ bildet aber $\tilde{N}_{0}$ eineindeutig auf die Menge aller Funktionen $N$ mit $\hat{N} \in \tilde{N}_{0}$ ab. Damit ist der Satz bewiesen.

\section{Sturm-Liouvillesche Differentialgleichungen mit indefiniter Gewichtsfunktion}

2.1. Wir betrachten in diesem Paragraphen die Anfangswertaufgabe

$$
\begin{gathered}
-\left(p(x) y^{\prime}(x)\right)^{\prime}+q(x) y(x)=\lambda r(x) y(x), \quad 0 \leqq x \leqq b, \\
p(0) y^{\prime}(0) \sin \alpha-y(0) \cos \alpha=0
\end{gathered}
$$

für ein $\alpha \in[0, \pi), \quad 0<b<\infty$, unter folgenden Voraussetzungen an die Koeffizientenfunktionen $p, q, r$ :

(I) $\quad p$ sei absolutstetig und positiv auf $[0, b]$;

(II) $q$ und $r$ seien summierbar und $q \geqq 0$ auf $[0, b]$ :

(III) für jedes $x$ mit $0<x<b$ gelte

$$
\int_{0}^{x}|r(s)| d s>0, \quad \int_{x}^{b}|r(s)| d s>0 .
$$

Die Lösung der Differentialgleichung (2.1a) mit den Anfangsbedingungen

$$
y(0)=\sin \alpha, \quad p(0) y^{\prime}(0)=\cos \alpha
$$

bezeichnen wir mit $u_{\alpha}(\cdot ; \lambda), 0 \leqq \alpha<\pi$.

Neben (2.1) betrachten wir die Randwertaufgabe

$$
-\left(p(x) w^{\prime}(x)\right)^{\prime}+q(x) w(x)=\lambda w(x), \quad 0 \leqq x \leqq b,
$$




$$
p(0) w^{\prime}(0) \sin \alpha-w(0) \cos \alpha=0, \quad w(b)=0 .
$$

Für $0 \leqq \alpha \leqq \pi / 2$ sind die Eigenwerte von (2.2) bekanntlich positiv; mit $\alpha_{0}$ bezeichnen wir denjenigen Wert von $\alpha$, für den der Nullpunkt Eigenwert von (2.2) ist. Dann gilt $\pi / 2<\alpha_{0}<\pi$, und

$$
|f(0)|^{2} \cot \alpha+\int_{0}^{b}\left(p(x)\left|f^{\prime}(x)\right|^{2}+q(x)|f(x)|^{2}\right) d x \geqq 0
$$

für alle stetig differenzierbaren Funktionen $f$ auf $[0, b]$ mit $f(b)=0$ und alle $\alpha \in\left(0, \alpha_{0}\right]$.

Der Einfachheit halber beschränken wir uns im folgenden auf Werte $\alpha \in\left(0, \alpha_{0}\right)$; die Randpunkte $\alpha=0$ oder $\alpha=\alpha_{0}$ erfordern eine Sonderbetrachtung (vgl. [3], § 14).

L e $\mathrm{m} m$ a 2.1. Es sei $\alpha \in\left(0, \alpha_{0}\right)$. Dann ist die Funktion $v_{\alpha}:=u_{\alpha}(\cdot ; 0)$ positiv auf $[0, b]$.

Beweis. Mit $\lambda_{\alpha}$ bezeichnen wir den kleinsten Eigenwert der Aufgabe (2.2) für das vorgegebene $\alpha \in\left(0, \alpha_{0}\right)$, mit $w_{\alpha}$ die zugehörige Eigenfunk. tion, $w_{\alpha}(0)=\sin \alpha$. Dann ist $w_{\alpha}$ positiv auf $[0, b)$ ([1], Satz 8.4.5). Die Differenz $v=v_{\alpha}-w_{\alpha}$ genügt der Differentialgleichung

$$
\left(p v^{\prime}\right)^{\prime}=q v+\lambda_{\alpha} w_{\alpha}
$$

und den Anfangsbedingungen

$$
v(0)=0, \quad p(0) v^{\prime}(0)=0 .
$$

Aus der Beziehung

$$
p(x) v^{\prime}(x)=\int_{0}^{x}\left(q(s) v(s)+\lambda_{\alpha} w_{\alpha}(s)\right) d s
$$

folgt, daß $p(x) v^{\prime}(x)$ für hinreichend kleines $x>0$ positiv, also die Funktion $v$ dort wachsend ist. Würde ein Punkt $x_{0}$ mit $p\left(x_{0}\right) v^{\prime}\left(x_{0}\right)=0$ existieren, so gäbe es einen kleinsten Punkt mit dieser Eigenschaft, woraus mit (2.3) leicht ein Widerspruch folgt.

Entsprechend wie in [3], § 14, transformieren wir (2.1) auf eine Aufgabe der Form (1.18) mit $D \equiv 0$. Zu diesem Zweck führen wir die neue unabhängige Variable

$$
X:=\int_{0}^{x} \frac{d s}{p(s) v_{\alpha}^{2}(s)}
$$


ein und ordnen den Funktionen $y$ auf $[0, b]$ Funktionen $Y$ auf $[0, l]$ mit

$$
l:=\int_{0}^{b} \frac{d s}{p(s) v_{\alpha}^{2}(s)}
$$

$\mathrm{zu}:$

$$
Y(X)=\frac{y(x)}{v_{\alpha}(x)},
$$

falls zwischen $x$ und $X$ die Beziehung (2.4) besteht; dabei hat $v_{\alpha}$ stets dieselbe Bedeutung wie in Lemma 2.1. Dann gilt

$$
Y^{\prime}(X)=v_{\alpha}(x) p(x) y^{\prime}(x)-y(x) p(x) v_{\alpha}^{\prime}(x),
$$

und die Aufgabe (2.1) ist äquivalent mit

$$
\begin{aligned}
\frac{d Y^{\prime}(X)}{d X}+\lambda Y(X) P(X) & =0, \quad 0 \leqq X \leqq l, \\
Y^{\prime}(0) & =0
\end{aligned}
$$

$\left(P(X):=r(x) p(x) v_{\alpha}^{4}(x)\right)$, d. h. mit (1.18) für

$$
d M(X)=P(X) d X, \quad D(X) \equiv 0 .
$$

Die Lösungen $\Phi(\cdot ; \lambda), \Psi(\cdot ; \lambda)$ von $(2.6)$ mit

$$
\begin{gathered}
\Phi(0 ; \lambda)=1, \quad \Phi^{\prime}(0 ; \lambda)=0, \\
\Psi(0 ; \lambda)=0, \quad \Psi^{\prime}(0 ; \lambda)=1
\end{gathered}
$$

Jassen sich folgendermaßen durch die Funktionen $u_{\alpha}(\cdot ; \lambda)$ ausdrücken:

$$
\Phi(X ; \lambda)=\frac{u_{\alpha}(x ; \lambda)}{v_{\alpha}(x)}, \quad \Psi(X ; \lambda)=\frac{u_{\alpha}(x ; \lambda) \cot \alpha-u_{\alpha+\pi / 2}(x ; \lambda)}{v_{\alpha}(x)} .
$$

2.2. Für eine stetig differenzierbare Funktion $y$ auf $[0, b]$ mit $y(b)=0$ ergibt sich mit der Transformation $F_{1}$ aus (1.23)

$$
\begin{aligned}
F_{1}^{\left[Y^{\prime \prime}\right]}(\lambda) & =\int_{0}^{l} Y(X) \Phi(X ; \lambda) P(X) d X \\
& =\int_{0}^{b} y(x) u_{\alpha}(x ; \lambda) r(x) d x=: F_{\alpha}^{[y]}(\lambda) .
\end{aligned}
$$


Defin ition 2.2. Es sei $\alpha \in\left(0, \alpha_{0}\right)$. Eine Funktion $\tau$ auf $(-\infty, \infty)$ heiße eine A-Spelctralfuntion der Anfangswertaufgabe (2.1), wenn sie die folgenden Eigenschaften hat:

(i) $\tau$ ist nichtfallend, $\tau(0)=0, \quad \tau(\lambda)=(\tau(\lambda+0)+\tau(\lambda-0)) / 2$ $(-\infty<\lambda<\infty)$;

(ii) für stetig differenzierbare Funktionen $y$ auf $[0, b]$ mit $y(b)=0$ gilt

$$
\begin{aligned}
& \int_{0}^{b}\left(p(x)\left|y^{\prime}(x)\right|^{2}+q(x)|y(x)|^{2}\right) d x+\cot \alpha|y(0)|^{2} \\
& =\int_{-\infty}^{\infty}\left|F_{\alpha}^{[y]}(\lambda)\right|^{2} d \tau(\lambda) .
\end{aligned}
$$

Die A-Spektralfunktion $\tau$ heißt orthogonal, wenn die Menge der Fouriertransformierten $F_{\alpha}^{[y]}$ für stetig differenzierbare Funktionen $y$ mit $y(b)=0$ in $L_{\tau}^{2}$ dicht liegt.

L e m m a 2.3. Jede A-Spektralfunktion (bzw. orthogonale A-Spektralfunktion) der Aufgabe (2.1) ist eine Spektralfunktion (bzw. orthogonale Spektralfunktion) der Aufgabe (2.6) (im Sinne der Definition 1.10).

Beweis. Es sei $\tau$ eine Spektralfunktion von (2.6). Dann gilt für stetig differenzierbare Funktionen $y$ mit $y(b)=0$

$$
\int_{0}^{l}\left|Y^{\prime}(X)\right|^{2} d X=\int_{-\infty}^{\infty}\left|F_{1}^{\left[Y^{\prime}\right]}(\lambda)\right|^{2} d \tau(\lambda),
$$

d. h. wegen $(2.7),(2.5),(2.4)$ und den Eigenschaften von $v_{\alpha}$

$$
\begin{aligned}
& \int_{-\infty}^{\infty}\left|F_{\alpha}^{[y]}(\lambda)\right|^{2} d \tau(\lambda)=\int_{0}^{b}\left|v_{\alpha}(x) p(x) y^{\prime}(x)-y(x) p(x) v_{\alpha}^{\prime}(x)\right|^{2} \frac{d x}{p(x) v_{\alpha}^{2}(x)} \\
= & \int_{0}^{b} p(x)\left|y^{\prime}(x)\right|^{2} d x-\int_{0}^{b} p(x) y^{\prime}(x) v_{\alpha}^{\prime}(x) \overline{y(x)} \frac{d x}{v_{\alpha}(x)} \\
& -\int_{0}^{b} \overline{y^{\prime}(x)} y(x) p(x) v_{\alpha}^{\prime}(x) \frac{d x}{v_{\alpha}(x)}+\int_{0}^{b}|y(x)|^{2} p(x) v_{\alpha}^{\prime}(x)^{2} \frac{d x}{v_{\alpha}^{2}(x)} \\
= & \int_{0}^{b} p(x)\left|y^{\prime}(x)\right|^{2} d x-\int_{0}^{b} p(x) v_{\alpha}^{\prime \prime}(x) \frac{d}{d x} \frac{|y(x)|^{2}}{v_{\alpha}(x)} d x
\end{aligned}
$$




$$
\begin{aligned}
& =\int_{0}^{b} p(x)\left|y^{\prime}(x)\right|^{2} d x-\left(\left.p(x) v_{\alpha}^{\prime}(x) \frac{|y(x)|^{2}}{v_{\alpha}(x)}\right|_{0} ^{b}+\int_{0}^{b}\left(p(x) v_{\alpha}^{\prime}(x)\right)^{\prime} \frac{|y(x)|^{2}}{v_{\alpha}(x)} d x\right. \\
& =\int_{0}^{b} p(x)\left|y^{\prime}(x)\right|^{2} d x+\cot \alpha|y(0)|^{2}+\int_{0}^{b} q(x)|y(x)|^{2} d x,
\end{aligned}
$$

d. h. $\tau$ ist eine $A$-Spektralfunktion der Aufgabe (2.1).

Umgekehrt ergibt sich für eine $A$-Spektralfunktion $\tau$ die Beziehung (2.8) für alle $Y^{\prime}$, die zu stetig differenzierbaren Funktionen $y$ mit $y(b)=0$ gehören. Die Menge dieser Funktionen liegt aber dicht in $L^{2}(0, l)$, daraus folgt die Behauptung.

Wir setzen im folgenden Satz

$$
\begin{aligned}
& V_{11}(\lambda):=1+\lambda \int_{0}^{b} u_{\alpha+\pi / 2}(x ; \lambda) u_{\alpha}(x ; 0) r(x) d x, \\
& V_{21}(\lambda):=-\lambda \int_{0}^{b} u_{\alpha}(x ; \lambda) u_{\alpha}(x ; 0) r(x) d x, \\
& V_{12}(\lambda):=-\lambda u_{\alpha+\pi / 2}(b ; \lambda), \\
& V_{22}(\lambda):=\lambda u_{\alpha}(b ; \lambda),
\end{aligned}
$$

sowie für $N \in \tilde{N}_{0}$ :

$$
\Omega_{N}(\lambda):=\lambda \cot \alpha+\lambda \frac{V_{11}(\lambda) N(\lambda)+V_{12}(\lambda)}{V_{21}(\lambda) N(\lambda)+V_{22}(\lambda)} .
$$

S a tz 2.4. Für $N \in \tilde{N}_{0}$ gehört die Funktion $\Omega_{N}$ zur Klasse $N_{0}$. Die Gesamtheit der A-Spektralfunktionen der Anfangswertaufgabe (2.1) stimmt mit der Gesamtheit der Funktionen $\tau_{N}$ :

$$
\tau_{N}(\lambda):=\frac{1}{\pi} \lim _{\varepsilon \downarrow 0} \int_{0}^{\lambda} \operatorname{Im} \Omega_{N}(\xi+i \varepsilon) d \xi
$$

überein, wenn $N$ die Klasse $\tilde{N}_{0}$ durchläuft. Dabei ergeben sich die orthogonalen A-Spektralfunktionen genau dann, wenn $N$ eine reelle Konstante (einschließlich $\infty$ ) ist.

Beweis. Wir gehen aus von der Beziehung

$$
\begin{aligned}
& \lambda \frac{\Psi_{-}^{\prime}(l ; \lambda) N(\lambda)+\lambda \Psi(l ; \lambda)}{\Phi_{-}^{\prime}(l ; \lambda) N(\lambda)+\lambda \Phi(l ; \lambda)}=\lambda \cot \alpha \\
& +\lambda \frac{\left(u_{\alpha+\pi / 2}(b ; \lambda) v_{\alpha}^{\prime}(b) p(b)-u_{\alpha+\pi t 2}^{\prime}(b ; \lambda) v_{\alpha}(b) p(b)\right) N(\lambda) v_{\alpha}(b)-\lambda u_{\alpha+\pi / 2}(b ; \lambda)}{\left(u_{\alpha}^{\prime}(b ; \lambda) v_{\alpha}(b) p(b)-u_{\alpha}(b ; \lambda) v_{\alpha}^{\prime}(b) p(b)\right) N(\lambda) v_{\alpha}(b)+\lambda u_{\alpha}(b ; \lambda)} .
\end{aligned}
$$


Weiter gilt

$$
\begin{aligned}
& u_{\alpha+\pi / 2}(x ; \lambda) v_{\alpha}^{\prime}(x) p(x)-u_{\alpha+\pi / 2}^{\prime}(x ; \lambda) v_{\alpha}(x) p(x) \\
& =1+\lambda \int_{0}^{x} u_{\alpha+\pi / 2}(s ; \lambda) v_{\alpha}(s) r(s) d s \\
& u_{\alpha}^{\prime}(x ; \lambda) v_{\alpha}(x) p(x)-u_{\alpha}(x ; \lambda) v_{\alpha}^{\prime}(x) p(x) \\
& =-\lambda \int_{0}^{x} u_{\alpha}(s ; \lambda) v_{\alpha}(s) r(s) d s
\end{aligned}
$$

woraus auf Grund von Satz 1.12 und Lemma 2.3 die Behauptung folgt.

Ist die Funktion $N$ insbesondere meromorph, so gilt dasselbe für $\Omega_{N}$; dann ist also $\tau_{N}$ eine Sprungfunktion. Die Sprünge von $\tau_{N}$ sind genau die Eigenwerte der Randwertaufgabe

$$
\begin{gathered}
-\left(p(x) y^{\prime}(x)\right)^{\prime}+q(x) y(x)=\lambda r(x) y(x), \quad 0 \leqq x \leqq b, \\
p(0) y^{\prime}(0) \sin \alpha-y(0) \cos \alpha=0, \quad p(b) y^{\prime}(b) N_{1}(\lambda)+y(b)=0
\end{gathered}
$$

mit

$$
N_{1}(\lambda)=\frac{N(\lambda) v_{\alpha}(b)}{\lambda-v_{\alpha}^{\prime}(b) p(b) N(\lambda)} .
$$

Insbesondere entspricht die Funktion $N$ :

$$
N(\lambda)=\tan \beta \frac{\lambda}{v_{\alpha}(b)+\tan \beta v_{\alpha}^{\prime}(b) p(b)}
$$

in (2.9) der Randbedingung

$$
p(b) y^{\prime}(b) \sin \beta+y(b) \cos \beta=0 .
$$

Diese Randbedingung erzeugt also für das vorgegebene $\alpha \in\left(0, \alpha_{0}\right)$ genau dann eine $A$-Spektralfunktion, wenn die Funktion $N$ aus (2.11) zur Klasse $N_{0}$ gehört, d. h. für

$$
\begin{aligned}
& 0 \leqq \beta \leqq \pi-\arctan \frac{v_{\alpha}(b)}{p(b) v_{\alpha}^{\prime}(b)} \quad \text { fälls } \quad v_{\alpha}^{\prime}(b)>0, \\
& 0 \leqq \beta \leqq-\arctan \frac{v_{\alpha}(b)}{p(b) v_{\alpha}^{\prime}(b)} \quad \text { falls } \quad v_{\alpha}^{\prime}(b)<0 .
\end{aligned}
$$

2.3. In diesem Abschnitt stellen wir an die Koeffizientenfunktionen $p, q, r$ zunächst nur die Bedingungen (I) und (II) aus Abschnitt 1. Wir betrachten den Weylschen Koeffizienten $l_{N_{1}}^{(\alpha)}$ : 


$$
l_{N_{1}}^{(\alpha)}(\lambda):=-\frac{p(b) u_{\alpha+\pi / 2}^{\prime}(b ; \lambda) N_{1}(\lambda)+u_{\alpha+\pi / 2}(b ; \lambda)}{p(b) u_{\alpha}^{\prime}(b ; \lambda) N_{1}(\lambda)+u_{\alpha}(b ; \lambda)} ;
$$

dabei sei $N_{1}$ eine außerhalb der reellen Achse stückweise meromorphe Funktion mit $N_{1}(\lambda)=\overline{N_{1}(\bar{\lambda})}$ oder $N_{1}(\lambda) \equiv \infty$. Da Zähler und Nenner in (2.12) nicht gleichzeitig verschwinden, ist $l_{N_{1}}^{(\alpha)}(\lambda)$ für alle nichtreellen $\lambda$ erklärt, wenn wir nur $\infty$ als möglichen Wert zulassen. Die Funktion $\chi_{N_{1}}(\cdot ; \lambda)$ :

$$
\begin{array}{ll}
\chi_{N_{1}}(x ; \lambda)=u_{\alpha+\pi / 2}(x ; \lambda)+l_{N_{1}}^{(\alpha)}(\lambda) u_{\alpha}(x ; \lambda) & \left(l_{N_{1}}^{(\alpha)}(\lambda) \neq \infty\right), \\
\chi_{N_{1}}(x ; \lambda)=u_{\alpha}(x ; \lambda) & \left(l_{N_{1}}^{(\alpha)}(\lambda)=\infty\right)
\end{array}
$$

genügt also der Differentialgleichung (2.1a) und der Randbedingung

$$
p(b) y^{\prime}(b) N_{1}(\lambda)+y(b)=0 .
$$

Analog wie in Abschnitt 1.4 multiplizieren wir im Falle $l_{N_{1}}^{(\alpha)}(\lambda) \neq \infty$, $l_{N_{1}}^{(\alpha)}(\mu) \neq \infty$ die Identität

$$
\begin{aligned}
& \int_{0}^{b}\left(p(x) \chi_{N_{1}}^{\prime}(x ; \lambda) \overline{\chi_{N_{1}}^{\prime}(x ; \mu)}+q(x) \chi_{N_{1}}(x ; \lambda) \overline{\left.\chi_{N_{1}}(x ; \mu)\right)} d x\right. \\
& =p(b) \chi_{N_{1}}(b ; \lambda) \overline{\chi_{N_{1}}^{\prime}(b ; \mu)}-p(0)\left(\cos \alpha+l_{N_{1}}^{(\alpha)}(\lambda) \sin \alpha\right)\left(\sin \alpha+\overline{l_{N_{1}}^{(\alpha)}(\mu)} \cos \alpha\right) \\
& \quad+\bar{\mu} \int_{0}^{b} r(x) \chi_{N_{1}}(x ; \lambda) \overline{\chi_{N_{1}}(x ; \mu)} d x
\end{aligned}
$$

mit $\lambda$ und subtrahieren davon die nach Vertauschung von $\lambda$ und $\bar{\mu}$ erhaltene Gleichung. Dann ergibt sich durch einfache Rechnung im Falle $\alpha \neq 0$ für $\tilde{l}_{N_{1}}^{(\alpha)}(\lambda):=l_{N_{1}}^{(\alpha)}(\lambda)+\cot \alpha:$

$$
\begin{aligned}
& p(0) \frac{\lambda \tilde{l}_{N_{1}}^{(\alpha)}(\lambda)-\bar{\mu} \tilde{l}_{N_{1}}^{(\alpha)}(\mu)}{\lambda-\bar{\mu}}=\frac{\sin 2 \alpha}{2} p(0) \tilde{l}_{N_{1}}^{(\alpha)}(\lambda) \overline{\tilde{l}_{N_{1}}^{(\alpha)}(\mu)} \\
& +\int_{0}^{b}\left(p(x) \chi_{N_{1}}^{\prime}(x ; \lambda) \overline{\chi_{N_{1}}^{\prime}(x ; \mu)}+q(x) \chi_{N_{1}}(x ; \lambda) \overline{\chi_{N_{1}}(x ; \mu)}\right) d x \\
& +p(b)^{2} \chi_{N_{1}}^{\prime}(b ; \lambda) \overline{\chi_{N_{1}}^{\prime}(b ; \mu)} \frac{\lambda N_{1}(\lambda)-\bar{\mu} \overline{N_{1}(\mu)}}{\lambda-\bar{\mu}}
\end{aligned}
$$

und im Falle $\alpha=0$ für $\check{l}_{N_{1}}^{(\alpha)}(\lambda):=\frac{1}{\lambda} l_{N_{1}}^{(\alpha)}(\lambda)$ :

$$
p(0) \frac{\check{l}_{N_{1}}^{(\alpha)}(\lambda)-\overline{\check{l}_{N_{1}}^{(\alpha)}(\mu)}}{\lambda-\bar{\mu}}
$$




$$
\begin{gathered}
=\frac{1}{\lambda \bar{\mu}}\left\{\int_{0}^{b}\left(p(x) \chi_{N_{1}}^{\prime}(x ; \lambda) \overline{\chi_{N_{1}}^{\prime}(x ; \mu)}+q(x) \chi_{N_{1}}(x ; \lambda) \overline{\chi_{N_{1}}(x ; \mu)}\right) d x\right. \\
\left.+p(b)^{2} \chi_{N_{1}}^{\prime}(b ; \lambda) \overline{\chi_{N_{1}}^{\prime}(b ; \mu)} \frac{\lambda N_{1}(\lambda)-\mu \overline{N_{1}(\mu)}}{\lambda-\bar{\mu}}\right\} .
\end{gathered}
$$

Damit ist der folgende Satz bewiesen.

S a tz 2.5. Gehört die Funktion $\hat{N}_{1}$ zur Klasse $\tilde{N}_{\varkappa^{\prime}}$ für einen Index $x^{\prime}, 0 \leqq x^{\prime}<\infty$, so gilt für $\alpha \in(0, \pi)$ :

und für $\alpha=0$ :

$$
\tilde{l}_{N_{1}}^{(\alpha)} \in \tilde{N}_{\varkappa}
$$

$$
l_{N_{1}}^{(\alpha)} \in N_{x}
$$

dabei genügt der Index $x$ den folgenden Ungleichungen:

$$
\begin{aligned}
& 0 \leqq x \leqq x^{\prime} \quad \text { für } \alpha \in\left[0, \frac{\pi}{2}\right], \\
& 0 \leqq x \leqq x^{\prime}+1 \quad \text { für } \alpha \in\left(\frac{\pi}{2}, \pi\right) .
\end{aligned}
$$

Es sei jetzt auch die Voraussetzung (III) aus Abschnitt 1 erfüllt. Dann folgt z. B. aus Satz 1.7 (b), daß die Funktion $l_{N_{1}}^{(\alpha)}$ nicht identisch unendlich ist, wenn $\hat{N}_{1}$ zu einer Klasse $\tilde{N}_{\varkappa^{\prime}}$ gehört. Gilt $\hat{N}_{1} \in \tilde{N}_{0}$ und $0<\alpha \leqq \pi / 2$, so ergibt sich für die gemäß (2.10) gebildete Funktion $N: N \in \tilde{N}_{0}$ (für $0<\alpha<\pi / 2$ ist $\left.v_{\alpha}^{\prime}(b) \geqq 0\right)$. Da außerdem offensichtlich die Beziehung

$$
\Omega_{N}(\lambda)=\lambda l_{N_{1}}^{(\alpha)}(\lambda)
$$

besteht, folgt dann die Aussage $\hat{l}_{N_{1}}^{(\alpha)} \in N_{0}$ auch aus Satz 2.4.

Für $N_{1} \equiv 0, \pi / 2<\alpha<\pi$, ergibt sich aus Satz 2.5 insbesondere, daß die Funktion $\hat{\imath}_{0}^{(\alpha)}$ einer Klasse $\tilde{N}_{\varkappa}$ mit $\varkappa=0$ oder $\varkappa=1$ angehört. Sichert man durch geeignete Voraussetzungen an $r$ (z. B. Voraussetzung (III) aus Abschnitt 1, vgl. auch [2]), daß sie nicht identisch $\infty$ ist, so hat sie nach den oben zitierten Eigenschaften der Funktionen der Klassen $\boldsymbol{N}_{\varkappa}$ höchstens einen e i n $\mathrm{f} \mathrm{a} \mathrm{ch}$ e $\mathrm{n}$ Pol in der oberen (oder unteren) Halbebene. Dann gilt dasselbe für den Weylschen Koeffizienten $l_{0}^{(\alpha)}$. Dieser stimmt aber mit dem in [2], S. 373 eingeführten Koeffizienten $l$ überein, also hat auch $l$ in der oberen (oder unteren) Halbebene höchstens einen einfachen Pol. Diese Eigenschaft überträgt sich leicht auf die in [2] betrachtete Funktion $m$, womit die in [2] im Anschluß an Satz 2 aufgeworfene Frage beantwortet ist. 


\section{Der Differentialausdruck $d f_{+}^{\prime}+\lambda f d M+\lambda^{2} f d D$ als 0peratorenschar}

3.1. Wir setzen jetzt

$$
\begin{gathered}
\mathfrak{D}(A):=\left\{f \in \mathfrak{D}_{0}: f_{-}^{\prime}(0)=0, f(l)=f_{-}^{\prime}(l)=0\right\}, \\
A f:=-\varphi,
\end{gathered}
$$

wenn $f$ die Darstellung (1.3) besitzt. Dann ist der Operator $A$ in $L_{V}^{2}$ symmetrisch, abgeschlossen und positiv. Es gilt nämlich für $f \in \mathscr{D}(A)$

$$
(A f, f)=\int_{0}^{l}\left|\int_{0}^{x} \varphi(t) d V(t)\right|^{2} d x \geqq 0,
$$

und im Falle des Gleichheitszeichens folgt $\int_{0}^{x} \varphi(t) d V(t)=0$ für fast alle $x \in[0, l]$, also $\int_{0}^{l} \varphi(t) \chi_{\Delta}(t) d V(t)=0$ für jedes Intervall $\Delta \subset[0, l]$, d. h. $\varphi=0$ in $L_{V}^{2}$ und somit $f \equiv 0$.

Mit den Operatoren $B$ und $C$ aus Abschnitt 1.2 betrachten wir die auf der (in $L_{V}^{2}$ dichten) Menge $\mathfrak{D}(A)$ definierte Schar $L$ :

$$
L(\lambda):=-A+\lambda B+\lambda^{2} C .
$$

Entsprechend einem allgemeinen Vorgehen in der Theorie der Operatorenscharen ordnen wir der Schar $L$ einen Operator $L$ in einem größeren Raum zu, dessen Spektraleigenschaften mit denen der Schar $L$ übereinstimmen.

Zu diesem Zweck versehen wir die Menge $L_{V}^{2} \oplus \mathscr{D}(A)$ mit dem Skalarprodukt

$$
\begin{gathered}
\{\boldsymbol{f}, \boldsymbol{g}\}:=\left(C f_{1}, g_{1}\right)+\left(A f_{2}, g_{2}\right), \\
\boldsymbol{f}=\left(\begin{array}{c}
f_{1} \\
f_{2}
\end{array}\right), \quad g=\left(\begin{array}{c}
g_{1} \\
g_{2}
\end{array}\right), \quad f_{1}, g_{1} \in L_{V}^{2} ; f_{2}, g_{2} \in \mathfrak{D}(A) .
\end{gathered}
$$

Dann gilt $\{\boldsymbol{f}, \boldsymbol{f}\} \geqq 0$, wobei das Gleichheitszeichen äquivalent ist mit $C f_{1}=0$ und $f_{2}=0$. Mit $\&$ bezeichnen wir den Faktorraum von $L_{V}^{2}$ $\oplus \mathscr{D}(A)$ nach der Menge aller $\boldsymbol{f}$ mit $\{\boldsymbol{f}, \boldsymbol{f}\}=0$ und mit $\mathfrak{Q}_{0}$ denjenigen Teilraum von $\mathbf{2}$, der sich bei der Faktorraumbildung als Bild von $\mathfrak{D}(A) \oplus$ $\mathfrak{D}(A)$ ergibt, d. h., jedes $f \in \mathbf{\Omega}_{0}$ hat mindestens einen Repräsentanten.

$$
\left(\begin{array}{l}
f_{1} \\
f_{2}
\end{array}\right)
$$

dessen Komponenten beide zu $\mathfrak{D}(A)$ gehören.

Im Raum $\mathbf{Q}$ definieren wir einen linearen Operator $\boldsymbol{L}$ auf $\mathbf{a}_{0}$ durch die Festsetzung, daß 


$$
L f=g \quad\left(g \in \mathbf{Q}, f \in \mathbf{\Omega}_{0}\right)
$$

äquivalent sei mit der Gleichung

$$
\left(\begin{array}{cc}
-B & A \\
I & 0
\end{array}\right) f=\left(\begin{array}{ll}
C & 0 \\
0 & I
\end{array}\right) g
$$

Diese Definition ist korrekt, denn $\boldsymbol{f} \in \mathbf{2}_{0},\{\boldsymbol{f}, \boldsymbol{f}\}=0$ ist gleichbedeutend mit $C f_{1}=0, f_{2}=0$. Beziehung (3.2) besagt aber $-B f_{1}=C g_{1}$, $f_{1}=g_{2}$, damit folgt $0=-B C f_{1}=C^{2} g_{1}$, also $C g_{1}=0, B f_{1}=0$ und auf Grund von (1.8) schließlich $g_{2}=f_{1}=|B| f_{1}+C f_{1}=0$.

Für $f \in \mathbf{\Omega}_{0}$ und $g=L f$ gilt weiter

$$
\begin{aligned}
\{\boldsymbol{L} \boldsymbol{f}, \boldsymbol{f}\} & =\{\boldsymbol{g}, \boldsymbol{f}\}=\left(C g_{1}, f_{1}\right)+\left(A g_{2}, f_{2}\right) \\
& =-\left(B f_{1}, f_{1}\right)+\left(A f_{2}, f_{1}\right)+\left(A f_{1}, f_{2}\right),
\end{aligned}
$$

also ist der Operator $L$ symmetrisch.

3.2. L e m m a 3.1. Die Abbildung $\Phi \mid \mathbf{R} \times C \rightarrow C$ :

$$
\Phi(f ; \lambda):=\frac{1}{\lambda} \int_{0}^{l} f_{2}(x) d \varphi_{+}^{\prime}(x ; \lambda)-\int_{0}^{l} f_{1}(x) \varphi(x ; \lambda) d D(x) \quad\left(f=\left(\begin{array}{l}
f_{1} \\
f_{2}
\end{array}\right)\right)
$$

ist ein richtendes Funktional für den Operator $\boldsymbol{L}$ in $\mathbf{2}$.

Beweis. Die Definition (3.3) ist korrekt, denn aus $\{\boldsymbol{f}, \boldsymbol{f}\}=0, \mathrm{~d} . \mathrm{h}$. $C f_{1}=0, f_{2}=0$, folgt $\Phi(f ; \lambda)=0$. Wir haben zu zeigen, daß für $\lambda \in C$ und $f \in \mathbf{\Omega}$ die Gleichung

$$
(L-\lambda I) g=f
$$

genau dann eine Lösung $\boldsymbol{g} \in \mathfrak{:}_{\mathbf{0}}$ hat, wenn $\Phi(f ; \lambda)=0$ gilt.

Die Beziehung (3.4) ist äquivalent den Gleichungen

$$
\begin{gathered}
d\left(g_{2}\right)_{+}^{\prime}+\lambda \beta g_{2} d V+\lambda^{2} \gamma g_{2} d V=-\left(\beta f_{2}+\gamma\left(f_{1}+\lambda f_{2}\right)\right) d V, \\
\left(g_{2}\right)_{-}^{\prime}(0)=0, \\
g_{1}=f_{2}+\lambda g_{2} .
\end{gathered}
$$

Bezeichnen wir die rechte Seite der Differentialgleichung aus (3.5) mit $h$, so ergibt sich (vgl. [3], Lemma 4.1) als allgemeine Lösung von (3.5)

$$
\begin{aligned}
& g_{2}(x)= \\
& \psi(x ; \lambda) \int_{0}^{x} h(s) \varphi(s ; \lambda) d V(s)+\varphi(x ; \lambda) \int_{x}^{l} h(s) \psi(s ; \lambda) d V(s)+c \varphi(x ; \lambda) .
\end{aligned}
$$


Die Lösung $g$ mit den Komponenten (3.7) und (3.6) gehört genau dann zu $\mathbf{2}_{0}$, wenn $g_{2}(l)=g_{2}^{\prime}(l)=0$ gilt, d. h. die Gleichungen

$$
\begin{gathered}
\psi(l ; \lambda) \int_{0}^{l} h(s) \varphi(s ; \lambda) d V(s)+c \varphi(l ; \lambda)=0, \\
\psi_{-}^{\prime}(l ; \lambda) \int_{0}^{l} h(s) \varphi(s ; \lambda) d V(s)+c \varphi_{-}^{\prime}(l ; \lambda)=0
\end{gathered}
$$

bestehen. Diese sind aber auf Grund von (1.16) äquivalent mit

$$
\int_{0}^{l} h(s) \varphi(s ; \lambda) d V(s)=c=0 \text {. }
$$

Nun gilt weiter

$$
\begin{aligned}
& \int_{0}^{l} h(x) \varphi(x ; \lambda) d V(x) \\
& =-\int_{0}^{l}\left(f_{2}(x) \varphi(x ; \lambda) d M(x)+\left(f_{1}(x)+\lambda f_{2}(x)\right) \varphi(x ; \lambda) d D(x)\right) \\
& =-\int_{0}^{l} f_{2}(x)(\varphi(x ; \lambda) d M(x)+\lambda \varphi(x ; \lambda) d D(x))-\int_{0}^{l} f_{1}(x) \varphi(x ; \lambda) d D(x) \\
& =\frac{1}{\lambda} \int_{0}^{l} f_{2}(x) d \varphi_{+}^{\prime}(x ; \lambda)-\int_{0}^{l} f_{1}(x) \varphi(x ; \lambda) d D(x)=\Phi(f ; \lambda),
\end{aligned}
$$

womit das Lemma bewiesen ist.

D efinition 3.2. Es sei $L$ ein symmetrischer Operator in einem linearen Raum $\mathbf{\&}$ mit nichtnegativ semidefinitem skalarprodukt $\{\boldsymbol{f}, \boldsymbol{g}\}$ $(\boldsymbol{f}, \boldsymbol{g} \in \mathbf{Q})$ und einem richtenden Funktional $\Phi$. Dann heißt jede auf $(-\infty, \infty)$ definierte Funktion $\tau$ mit den Eigenschaften

(a) $\tau$ ist nichtfallend, $\tau(0)=0, \quad \tau(\lambda)=(\tau(\lambda+0)+\tau(\lambda-0)) / 2$ $(-\infty<\lambda<\infty)$;

$$
\text { (b) }\{\boldsymbol{f}, \boldsymbol{g}\}=\int_{-\infty}^{\infty} \Phi(\boldsymbol{f} ; \lambda) \overline{\Phi(\boldsymbol{g} ; \lambda)} d \tau(\lambda) \quad \text { für alle } \boldsymbol{f}, \boldsymbol{g} \in \mathbf{Q}
$$

eine Spektralfunktion des Operators $L$.

$\mathrm{Satz}$ 3.3. Die Spektralfunktionen der Anfangswertaufgabe (1.18) sind genau die Spektralfunktionen des gemäß (3.1) und (3.2) erklärten Operators $L$ in $\mathbf{Q}$. 
Beweis. Die Bedingung (b) aus Definition 3.2 besagt mit den in Abschnitt 1.5 eingeführten Transformationen $F_{1}$ und $F_{2}$ :

$$
\begin{aligned}
(A f, f) & =\int_{0}^{l}\left|f_{+}^{\prime}(x)\right|^{2} d x=\int_{-\infty}^{\infty}\left|F_{1}^{\left[f^{\prime}{ }^{]}\right]}(\lambda)\right|^{2} d \tau(\lambda) \quad(f \in \mathfrak{D}(A)), \\
(C g, g) & =\int_{0}^{l}|g(x)|^{2} d D(x)=\int_{-\infty}^{\infty}\left|F_{2}^{[g]}(\lambda)\right|^{2} d \tau(\lambda) \quad\left(g \in L_{V}^{2}\right), \\
0 & =\int_{-\infty}^{\infty} F_{1+}^{\left[f^{\prime}\right]}(\lambda) \overline{F_{2}^{[g]}(\lambda)} d \tau(\lambda) \quad\left(f \in \mathfrak{D}(A), g \in L_{V}^{2}\right) .
\end{aligned}
$$

Wie in Abschnitt 1.5 sieht man leicht, daß (3.9) (bzw. (3.11)) äquivalent ist $\mathrm{zu}$ (1.24) (bzw. (1.26)); die Beziehungen (3.10) und (1.25) sind offensichtlich äquivalent. Damit ist der Satz bewiesen.

Liegt die Menge $\mathbf{2}_{0}$ quasidicht in $\mathbf{2}, \mathrm{d}$. h., gibt es zu jedem $\boldsymbol{f} \in \mathbf{Q}$ eine Folge $\left(\boldsymbol{f}_{n}\right) \subset \mathbf{\Omega}_{0}$ mit $\left\{\boldsymbol{f}_{n}-\boldsymbol{f}, \boldsymbol{f}_{n}-\boldsymbol{f}\right\} \rightarrow 0$ für $n \rightarrow \infty$, so folgt die Existenz von Spektralfunktionen des Operators $L$ auch aus einem allgemeinen Satz von M. G. Krein [4]. Die Menge $\mathbf{2}_{0}$ ist insbesondere quasidicht in $\mathbf{2}$, wenn mit einem $\gamma_{0}>0$ gilt: $C \geqq \gamma_{0} I$, d. h. $\gamma(x) \geqq \gamma_{0}$ für $V$-fast alle $x \in[0, l]$.

3.3. Es sei jetzt $M \neq 0$. Dann gibt es ein Element $f_{0} \in \mathfrak{D}(A)$ mit $\int_{0}^{l} f_{0}(x) d M(x)=1$ : Anderenfalls wäre nämlich $\int_{0}^{l} f(x) \beta(x) d V(x)=0$ für alle $f \in \mathfrak{D}(A)$, also $\beta=0$ in $L_{V}^{2}$ und somit $M \equiv 0$.

Wir setzen in diesem Abschnitt für $f \in \mathfrak{I}(A): F^{[f]}:=F_{1}^{\left[f_{+}^{\prime}\right]}$, d. h. es ist

$$
F^{[f]}(\lambda):= \begin{cases}-\frac{1}{\lambda} \int_{0}^{l} f(x) d \varphi_{+}^{\prime}(x ; \lambda) & (\lambda \neq 0) \\ +\int_{0}^{l} f(x) d M(x) & (\lambda=0) .\end{cases}
$$

Für beliebiges $\boldsymbol{f} \in \mathbf{\Omega}$ der Gestalt $f=\left(\begin{array}{l}0 \\ f\end{array}\right)$ bilden wir das Element

$$
\hat{f}:=\left(\begin{array}{l}
0 \\
\hat{f}
\end{array}\right):=\left(\begin{array}{c}
0 \\
f-F^{[f]}(0) f_{0}
\end{array}\right) \in \mathbf{\Omega} .
$$

Dann gilt $\int_{0}^{l} \hat{f} d M=0$, und man sieht leicht, daß die Gleichung $\boldsymbol{L} \boldsymbol{h}=\hat{\boldsymbol{f}}$ die (eindeutig bestimmte) Lösung 


$$
\boldsymbol{h}=\left(\begin{array}{l}
h_{1} \\
h_{2}
\end{array}\right) \in \mathbf{\Omega}_{0}
$$

mit

$$
h_{1}=\hat{f}, \quad h_{2}(x)=\int_{0}^{l} s \hat{f}(s) d M(s)-\int_{0}^{x}(x-s) \hat{f}(s) d M(s)
$$

hat. Somit erhalten wir

$$
\{\boldsymbol{h}, \boldsymbol{f}\}=(B \hat{f}, f)=(B f, f)-F^{[f]}(0)\left(B f_{0}, f\right)
$$

und

$$
\begin{aligned}
\Phi(\boldsymbol{h} ; \lambda) & =\frac{1}{\lambda} \Phi(\hat{\boldsymbol{f}} ; \lambda)=\frac{1}{\lambda} F^{[\hat{f}]}(\lambda) \\
& =\frac{1}{\lambda}\left(F^{[f]}(\lambda)-F^{[f]}(0) F^{\left[f_{0}\right]}(\lambda)\right) .
\end{aligned}
$$

Folglich ergibt sich aus (3.8)

$$
\begin{aligned}
& \int_{0}^{l}|f(x)|^{2} d M(x)=(B f, f) \\
& =F^{[f]}(0)\left(B f_{0}, f\right)+\int_{-\infty}^{\infty}\left(F^{[f]}(\lambda)-F^{[f]}(0) F^{\left[f_{0}\right]}(\lambda)\right) F^{[f]}(\lambda) \frac{d \tau(\lambda)}{\lambda},
\end{aligned}
$$

womit der folgende Satz bewiesen ist.

S a t z 3.4. Es sei $M \neq 0$. Dann gilt für jede Spektralfunktion $\tau$ der Anfangswertaufgabe (1.18) nach Wahl eines Elementes $f_{0} \in \mathfrak{D}(A)$ mit $F^{\left[f_{0}\right]}(0)=1$ für beliebige $f, g \in \mathfrak{D}(\mathrm{A})$

$$
\begin{gathered}
\int_{0}^{l} f(x) \overline{g(x)} d M(x)=F^{[f]}(0) \int_{0}^{l} f_{0}(x) \overline{g(x)} d M(x) \\
+\int_{-\infty}^{\infty}\left(F^{[f]}(\lambda)-F^{[f]}(0) F^{\left[f_{0}\right]}(\lambda)\right) \overline{F^{[g]}(\lambda)} \frac{d \tau(\lambda)}{\lambda} .
\end{gathered}
$$

Hat $f \in \mathfrak{D}(A)$ insbesondere die Eigenschaft $\int_{0}^{l} f d M=0$, so erhält (3.12) die Form

$$
\int_{0}^{l} f(x) \overline{g(x)} d M(x)=\int_{-\infty}^{\infty} F^{[f]}(\lambda) \overline{F^{[g]}(\lambda)} \frac{d \tau(\lambda)}{\lambda} .
$$


Durch die Beziehungen (3.9), (3.10) und (3.12) sind alle drei mit den Operatoren $A, B$ und $C$ der Schar $L$ assoziierten Skalarprodukte mittels der Spektralfunktion dargestellt.

\section{Literatur}

[1] Atrkinson, F. V.: Discrete and continuous boundary problems. - Mathematics in Science and Engineering 8, Academic Press, New York-London, 1964.

[2] Atkinson, F. V., W. N. Everitrt and K. S. OnG: On the m-coefficient of Weyl for a differential equation with an indefinite weight function. - Proc. London Math. Soc. (3) 29, 1974, 368-384.

[3] КАC, I. S., und M. G. KREIN [И. С. Кац und М. Г. Крейн]: О спектральных функциях струны. - Anhang II (S. 648-737) zur russischen Übersetzung von [1]: Ф. Аткинсон, Дискретные и непрерывные граничные задачи, Издательство «Мир», Москва, 1968. Übersetzung: On the spectral functions of the string. - Amer. Math. Soc. Transl. (2) 103, 1974, $19-102$.

[4] KREĬN, M. G. [M. Г. Крейн]: Про ермітові оператори 3 напрямними функціоналами. - Zbirnik Prac' Inst. Mat. Akad. Nauk URSR 10, 1948, 83- 106.

[5] KREĬL, M. G., und H. LANGER [M. Г. Крейн und Г. К. Лангер]: О спектральной функции самосопряжённого оператора в пространстве с индефинитной метрикой. - Dokl. Akad. Nauk SSSR 152, 1963, 39-42. Übersetzung: The spectral function of a selfadjoint operator in a space with indefinite metric. - Soviet Mat. Dokl. 4, 1963, 1236-1239.

[6] - - - - U Über einige Fortsetzungsprobleme, die eng mit hermiteschen Operatoren im Raume $\Pi_{\varkappa}$ zusammenhängen. Teil I: Einige Funktionenklassen und ihre Darstellungen. - Math. Nachr. (erscheint demnächst).

[7] KÜHNE, R.: Minimaxprinzipe für stark gedämpfte Scharen. - Acta Sci. Math. (Szeged) 29, 1968, 39-68.

[8] LANGER, H.: Zur Spektraltheorie verallgemeinerter gewöhnlicher Differentialoperatoren zweiter Ordnung mit einer nichtmonotonen Gewichtsfunktion. - Universität Jyväskylä, Mathematisches Institut, Bericht 14, Jyväskylä, 1972, 1-58.

[9] Nevanlinna, R.: Asymptotische Entwicklungen beschränkter Funktionen und das Stieltjessche Momentenproblem. - Ann. Acad. Sci. Fenn. Ser. A. 18: $5,1922,1-53$.

[10] - - Ưber metrische lineare Räume. II. Bilinearformen und Stetigkeit. - Ann. Acad. Sci. Fenn. Ser. A. I. Math.-Phys. 113, 1952, 1-9.

[11] - - Über metrische lineare Räume. III. Theorie der Orthogonalsysteme. - Ann. Acad. Sci. Fenn. Ser. A. I. Math.-Phys. 115, 1952, 1-27.

[12] - - Erweiterung der Theorie des Hilbertschen Raumes. - Medd. Lunds Univ. Mat. Sem. Supplementband tillägnat Mareel Riesz. Tome supplémentaire dédié à Marcel Riesz, 1952, 160-168.

[13] - - Über metrische lineare Räume. IV. Zur Theorie der Unterräume. - Ann. Acad. Sci. Fenn. Ser. A. I. Math.-Phys. 163, 1954, 1-16.

[14] -»- Über metrische lineare Räume. V. Relationen zwischen verschiedenen Metriken. - Ann. Acad. Sci. Fenn. Ser. A. I. Math.-Phys. 222, 1956, $1-6$. 
[15] PESONEN, E.: Über die Spektraldarstellung quadratischer Formen in linearen Räumen mit indefiniter Metrik. - Ann. Acad. Sci. Fenn. Ser. A. I. Math. Phys. 227, 1956, 1-31.

[16] PLEIJEL, $\AA$.: A survey of spectral theory for pairs of ordinary differential operators. - Spectral theory and differential equations [Symposium, Dundee, 1974], Lecture Notes in Mathematics 448, Springer-Verlag, Berlin-Heidelberg-New York, 1975, 256-272.

[17] WERNER, B.: Das Spektrum von Operatorscharen mit verallgemeinerten Rayleighquotienten. - Arch. Rational Mech. Anal. 42, 1971, 223-238.

Technische Universität Dresden

Sektion Mathematik

DDR 8027 Dresden

Deutsche Demokratische Republik

Eingegangen am 23. September 1975 